This item was submitted to Loughborough's Research Repository by the author.

Items in Figshare are protected by copyright, with all rights reserved, unless otherwise indicated.

\title{
Multiscale modeling of single-phase multicomponent transport in the cathode gas diffusion layer of a polymer electrolyte fuel cell
}

PLEASE CITE THE PUBLISHED VERSION

http://dx.doi.org/10.1021/ef100190c

\section{PUBLISHER}

(c) American Chemical Society

\section{VERSION}

AM (Accepted Manuscript)

\section{PUBLISHER STATEMENT}

This work is made available according to the conditions of the Creative Commons Attribution-NonCommercialNoDerivatives 4.0 International (CC BY-NC-ND 4.0) licence. Full details of this licence are available at: https://creativecommons.org/licenses/by-nc-nd/4.0/

\section{LICENCE}

CC BY-NC-ND 4.0

\section{REPOSITORY RECORD}

Rama, Pratap, Yu Liu, Rui Chen, Hossein Ostadi, Kyle Jiang, Yuan Gao, Xiaoxian Zhang, Rosemary Fisher, and Michael Jeschke. 2015. "Multiscale Modeling of Single-phase Multicomponent Transport in the Cathode Gas Diffusion Layer of a Polymer Electrolyte Fuel Cell”. figshare. https://hdl.handle.net/2134/18545. 


\title{
Multi-Scale Modelling of Single-Phase Multi-
}

\section{Component Transport in the Cathode Gas Diffusion}

\section{Layer of a Polymer Electrolyte Fuel Cell}

\author{
Pratap Rama ${ }^{1,2}$, Yu Liu ${ }^{1}$, Rui Chen ${ }^{1}$, Hossein Ostadi ${ }^{3}$, Kyle Jiang ${ }^{3}$, Yuan Gao ${ }^{4}$, Xiaoxian Zhang ${ }^{4}$, \\ Rosemary Fisher ${ }^{5}$, Michael Jeschke
}

\begin{abstract}
\end{abstract}
This research reports a feasibility study into multi-scale polymer electrolyte fuel cell (PEFC) modelling through the simulation of macroscopic flow in the multi-layered cell via $1 \mathrm{D}$ electrochemical modelling, and the simulation of microscopic flow in the cathode gas diffusion layer (GDL) via 3D single-phase multi-component lattice Boltzmann (SPMC-LB) modelling. The heterogeneous porous geometry of the carbon-paper GDL is digitally reconstructed for the SPMC-LB model using X-ray computer micro-tomography. Boundary conditions at the channel and catalyst layer interfaces for the SPMC-LB simulations such as specie partial pressures and through-plane flow rates are determined using the validated 1D electrochemical model, which is based on the general transport equation (GTE) and volume-averaged structural properties of the GDL. The calculated pressure profiles from the two models are cross-validated to verify the SPMC-LB technique. The simulations reveal a maximum difference of $2.4 \%$ between the thickness-averaged pressures calculated by the two techniques, which is attributable to the actual heterogeneity of the porous GDL structure.

\footnotetext{
${ }^{1}$ Department of Aeronautical and Automotive Engineering, Loughborough University, Leicestershire LE11 3TU, United Kingdom

${ }^{2}$ Corresponding author: p.rama@lboro.ac.uk

${ }^{3}$ Department of Mechanical Engineering, University of Birmingham, Birmingham B15 2TT, United Kingdom

${ }^{4}$ Department of Engineering, University of Liverpool, Liverpool L69 3GQ, United Kingdom

5 Technical Fibre Products Ltd., Kendal LA9 6PZ, United Kingdom
} 


\section{Keywords}

Lattice Boltzmann, General Transport Equation, Multi-Component, Polymer Electrolyte Fuel Cell, Gas Diffusion Layer, Porous Flow Simulation

\section{Introduction}

The polymer electrolyte fuel cell (PEFC) is an energy conversion device which is set to play a central role in the portfolio of future energy technologies for a range of civil and military applications. It is potentially a zero-emissions technology which can operate silently and without intermediate thermal or mechanical energy conversion processes. Unlike a heat engine therefore, the maximum thermodynamic efficiency of the PEFC is not bound by the Carnot limit.

The current priority for PEFC research and development is to optimise the technology for cost, performance and reliability. In order to do so it is paramount to have a structured understanding of the electrochemical and fluidic transport processes that occur within its different layers under fuel cell operating conditions. Due to the opaque nature of the materials used in the PEFC and the length scales involved, in-situ measurement and characterisation is generally formidable. As such, mechanistic PEFC modelling based on volume-averaged modelling has as such largely assumed this mantle since the early 1990s [1,2].

The literature demonstrates that there are mainly three groups of mechanistic treatments to simulate transport across the PEFC; electrochemical treatments, computational fluid dynamic (CFD) treatments and porous flow treatments. The first two of these are based on volume-averaged approaches that do not capture the actual geometry of porous fuel cell layers. They provide a valuable insight into the general behaviour of multi-component and multi-phase transport through the PEFC but cannot 
elucidate its dependence on the actual heterogeneous geometry of porous structures or the actual properties of its internal surfaces.

In the authors' previous research, a single-phase single component (SPSC) LB method was applied to simulate and validate transport through a representative model of a porous fuel cell gas diffusion layer (GDL) which was digitally reconstructed using X-ray micro-tomography [25]. In another piece of previous research, the authors developed, validated and applied an electrochemical model of multi-component and multi-phase transport through the complete multi-layered fuel cell [4].

The purpose of this research is to amalgamate the electrochemical model of the multi-layer PEFC with a newly-developed multi-component form of the single-phase LB model. The multi-layer PEFC model will be employed to determine the thermo-fluidic boundary conditions of the GDL for the LB model, and the LB model will be applied to determine the distribution of a multi-component gas through a representative three-dimensional structure of a GDL reconstructed using X-ray tomography. The aim of this research is to establish a multi-scale modelling treatment that has the potential to elucidate the effect of representative fuel cell operating conditions and representative structural and material properties of porous fuel cell materials on micro-scale internal transport. While the current study focuses on single-phase transport, it is envisaged that the modelling principles established here will be applied to study two-phase phenomena in subsequent research to capture the relevant physics of the system more completely. As such, it is anticipated that the multi-scale modelling approach developed herein will enable a step-change in the development of PEFC technology in terms of cost, performance and reliability.

\section{$2 \quad$ Literature Review}


As mentioned, mechanistic PEFC models can generally be split up into three different types of treatments; electrochemical, fluid dynamic and porous flow. The following literature review provides a brief discussion of each treatment, their capabilities and their limitations.

\section{Electrochemical Treatments}

Electrochemical treatments are generally based on dilute solution theory or concentrated solution theory and describe the transport characteristics of potentially multi-phase, multi-component flows through porous and quasi-porous regions of the multi-layered cell $[3,4]$. The detailed electrochemical treatment usually limits the mathematical treatment to one-dimension (through the thickness of the cell) [5,6] or two-dimensions (through-the thickness of the cell and along the channel length) $[7,8,9,10,11]$. A distinction between porous and quasi-porous materials can be made by considering layers such as the gas diffusion layer (GDL) and the polymer electrolyte membrane (PEM). The GDL is a carbon-fibre based material which has a compressible porous structure. The PEM on the other hand is impermeable to gases but by virtue of its partially hydrophobic and partially hydrophilic nature can behave like a porous material when liquid water infiltrates the material and forcibly expands its internal pores. To accommodate this type of behaviour where the structure of the material changes according to the operating conditions of the cell, it is necessary to employ the aforementioned electrochemical treatments.

Electrochemical treatments are well-suited to generating an understanding of multi-phase transport and cross-flow through multiple layers of the cell [4], the effects of porous layer compression [12], effects of PEM water uptake including Schroeder's paradox [13], PEM expansion and PEM constraint [14], water vapour transport [11] and the effect of highly wet-proofed assemblies on cell performance such as the microporous layer (MPL) [15]. Electrochemical treatments cannot detail the 
movement of individual components or phases through actual porous geometries of PEFC structures on their own.

\section{Computational Fluid Dynamic Treatments}

Computational fluid dynamic (CFD) treatments also simulate the movement of potentially multicomponent and multi-phase through porous and non-porous layers of the cell using equations of mass, momentum and energy conservation. CFD treatments are readily applied to three-dimensional nonporous geometries of the PEFC such as bipolar plate (BPP) flow-fields, and inlet and exit manifolds of PEFC stacks to simulate reactant transport and product removal. By assuming volume-averaged properties of porous layers such as porosity and tortuosity, CFD models can also be applied to simulate bulk transport through layers such as the GDL. They can also be incorporated with additional source and sink terms to account for electrode-kinetics to simulate reactant consumption and product generation in the catalyst layers (CL). CFD treatments command the use of fixed-geometry meshes for the non-porous and porous regions of the cell. Therefore, CFD treatments alone cannot readily deal with the compressibility of porous regions nor can they account for electrochemical transport across quasi-porous layers such as the PEM. To deal with cross-flow across the PEM - which inevitably couples and controls the composition of the gases in the anode and cathode sides of the cell - CFD treatments have to be incorporated with electrochemical treatments, such as those described previously.

CFD treatments are well suited to simulating three-dimensional flow characteristics through complex non-porous structures such as the flow fields of a fuel cell bi-polar plate (BPP) [16], flow through porous regions on a volume-averaged basis $[17,18,19]$ and planar temperature and current density distributions across the surface of the PEFC [20,21]. CFD treatments are not well-suited to simulating electrochemical cross-flow through quasi-porous layers, effects of layer 
compression/expansion/constraint as this invokes dynamic boundaries, or again flow through actual heterogeneous porous structures of PEFC layers

\section{Porous Flow Treatments}

Porous flow treatments can potentially take into account the actual porous structure of a given porous structure and simulate the movement of multi-component, multi-phase flows through its tortuous porous network. Unlike the previous two treatments, this method provides a visual and numerical understanding of the relationship between micro- and nano-scopic structure and porous flow, which can assist in optimising material design and cell operation. The lattice-Boltzmann method is a promising technique for porous flow simulation. It is based on kinetic theory and calculates the spatio-temporal redistribution of fictitious gaseous particles in a lattice based on their movement and collisions which conserve mass and momentum. . It is possible to employ this technique using representative threedimensional digital models of the porous structure that are reconstructed using stochastic software-based techniques [22,23] or X-ray tomography [24].

The lattice-Boltzmann technique is well-suited to simulating the movement of individual components and phases through the porous network of a representative structure as part of a multicomponent and multi-phase system, the effect of surface hydrophobicity on internal transport, reactive flow simulation and simulating transport in systems with dynamic structural boundaries. The latticeBoltzmann technique is not well-suited to simulating flow across quasi-porous layers or simultaneous multi-layer PEFC transport.

Clearly, the different types of modelling treatments discussed above can satisfy different objectives for PEFC technology development in terms of material composition and design, and cell 
configuration and operation. To date, much of the work in the literature has focused on electrochemical treatments [2] and CFD treatments [1]. However, in order to enable a step-change in the understanding of flow through multi-component and potentially multi-phase flow through porous PEFC media, the actual geometry of a porous structure has to be accounted for.

\section{$3 \quad$ Methodology}

In this research, the electrochemical model developed previously by the authors is applied to simulate multi-component mass transport across a multi-layered PEFC in one-dimension. The resulting partial pressures that are calculated at the cathode GDL/channel interface perpendicular to the direction of flow and species flow rates are supplied to the newly-developed SPMC-LB model as boundary conditions in order to simulate the distribution and movement of individual components through the porous structure of the cathode GDL. In applying the LB model, a representative three-dimensional model of the GDL that is digitally reconstructed using X-ray micro-tomography is employed in order to capture the actual geometric features of the porous material, as manufactured.

The current treatment will be limited to simulating single-phase flow and will not currently consider the deformation of the porous GDL structure as a result of compression. Both catalyst layer will be treated as infinitely thin interfaces between the GDL and PEM.

In order to apply the electrochemical model, certain material-specific properties will need to be determined, i.e., material porosity, and tortuosity and gas-phase permeability in the principal flow direction. These parameters will be deduced directly by applying the existing SPSC-LB model to the digital model of the GDL [25]. The electrochemical model will determine the amount of oxygen and water travelling through the cathode GDL and the partial pressures of the gas components (oxygen, 
nitrogen and water) at the interface between the cathode GDL and the cathode channel based on a macro-homogeneous treatment of the porous layers. These boundary conditions will be determined as a function of the operating current density, the conditions of both reactant supplies, the cross-flow of water through the PEM and the material properties of the PEFC layers such as those for the porous GDL mentioned above and the PEM including thickness, equivalent weight and dry density. Figure 1 illustrates the multi-scale modelling domain of the current study. Figure 2 illustrates the boundary conditions that are specified by the electrochemical model for the SPMC-LB model. Figure 3 demonstrates the overall multi-scale modelling process for the current study.

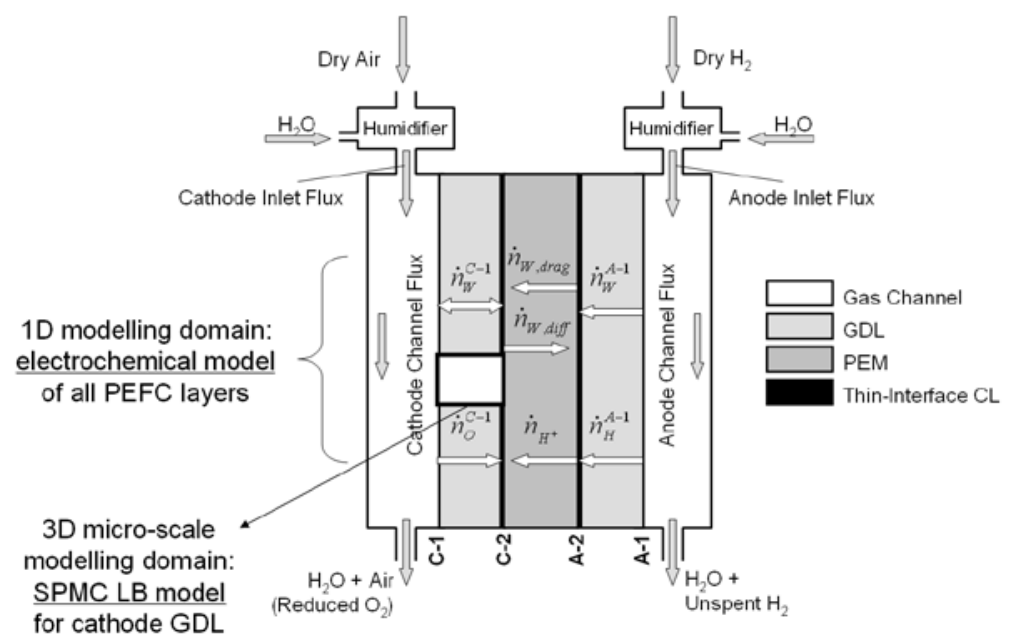

Figure 1. Multi-scale modelling domain for the current study

In order to cross-validate the predicted pressure profiles of oxygen, nitrogen and water vapour through the cathode GDL, the calculated results from the macroscopic treatment of the electrochemical model will be compared to those from the microscopic treatment of the lattice Boltzmann model, which is based on the actual heterogeneous geometry of the carbon paper GDL. The purpose of the crossvalidation is to demonstrably verify the newly developed SPMC-LB technique and to assess its capability to predict flow in heterogeneous porous structures in comparison to a macro-homogeneous treatment. 


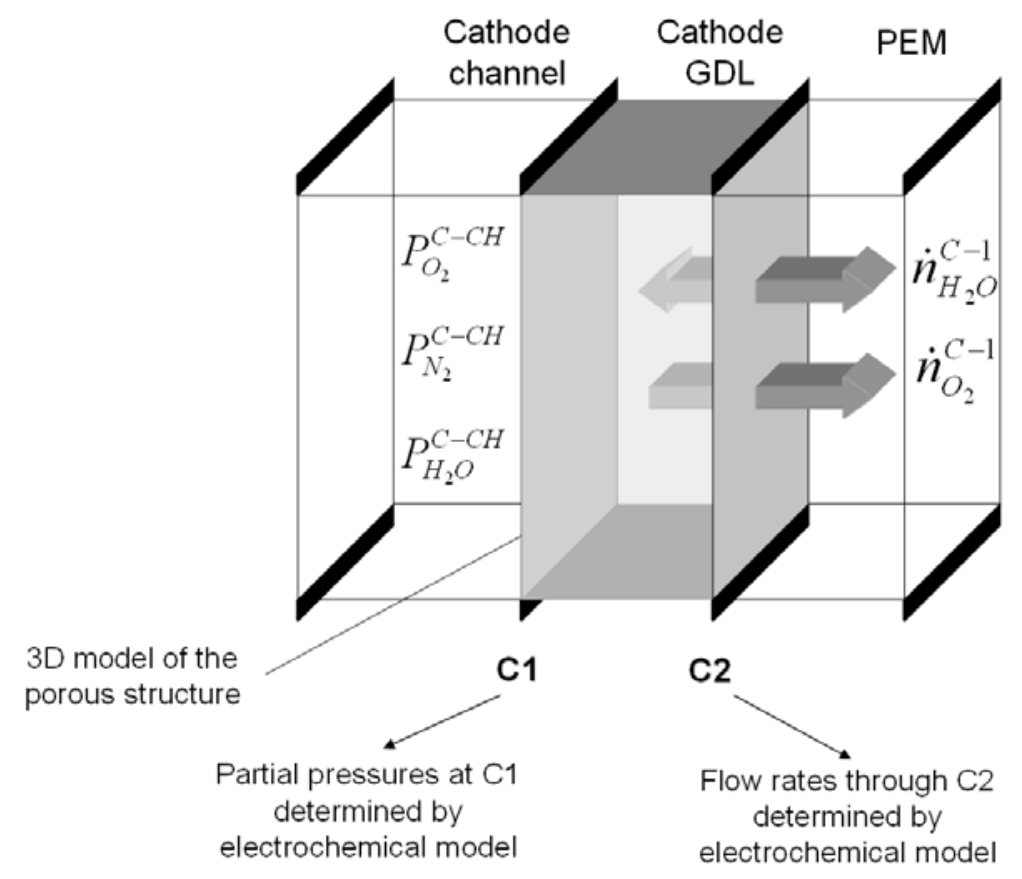

Figure 2 Three-dimensional micro-scale modelling domain for SPMC LB simulations of the cathode GDL with boundary conditions from the 1D electrochemical model

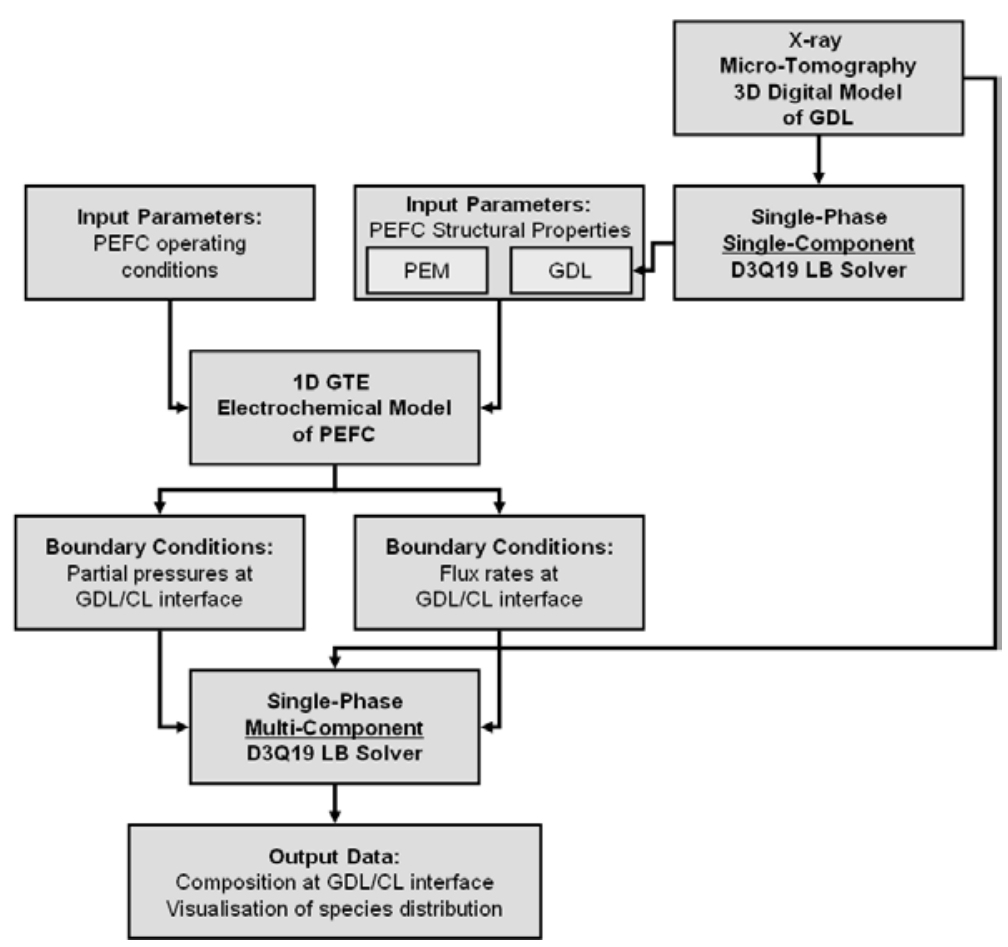

Figure 3 Overall methodology for the current study 
The electrochemical model applied in the current study was previously validated against experimental water transport data obtained from water balance measurements, magnetic resonance imaging (MRI) of water uptake across the PEM and embedded potential probe measurements of PEM conductivity [4]. The numerical foundation of the single-phase lattice-Boltzmann method employed in the current work was validated previously in single-component form by comparing the calculated permeability of a material sample using pressure drop measurements against the simulated permeability of the same sample using a digitally reconstructed model of its porous structure [25].

\section{$4 \quad$ Numerical Simulation}

The modelling description is presented here in three parts. The first part discusses the key equations in the new 3D single-phase multi-component LB model. The second part discusses the key equations of the electrochemical model and the application of the general transport equation (GTE) to describe multi-component, multi-phase flow through the layers of a PEFC. The final part discusses the $\mathrm{X}$-ray micro-tomography technique and the process by which a representative three-dimensional digital model of an actual GDL sample is generated.

\subsection{Single-Phase Multi-Component Lattice-Boltzmann Model}

\section{Key Equations for the Lattice-Boltzmann Model}

The movement of each gas species through the void space of the digital GDL model is simulated by the lattice Boltzmann equation (LBE) method. The LBE model is a numerical method that has been developed over the last decade to simulate fluid dynamics. Unlike traditional CFD methods, LBE 
models do not directly solve a set of partial differential equations. Instead, it simulates fluid flow by tracking the movement and collision of a number of fictitious particles under rules that ensure that collisions conserve mass and momentum.

Several LB methods has been developed over the past few years to simulate multiple-component gas transport through porous geometries, most notably for solid oxide fuel cell (SOFC) modelling $[26,27,28]$. The method used in this paper is based on that proposed by Luo et al. [29], which has been designed to simulate the dynamics of a two-component system. In this paper, we extended it to three components. The movement and collision of the particles of each species are described by the following lattice Boltzmann equation [27]:

$$
f_{k}^{i}\left(\boldsymbol{x}+\xi_{\boldsymbol{k}}^{i} \delta t, t+\delta t\right)=f_{k}^{i}(\boldsymbol{x}, t)+Q_{k}^{i i}(\boldsymbol{x}, t)+\sum_{j \neq i} Q_{k}^{i j}(\boldsymbol{x}, t)
$$

where $f_{k}^{i}(\mathrm{x}, t)$ is the mass of particle of species $i$ at location $\boldsymbol{x}$ and at time $t$, moving with velocity $\xi_{k}^{i}$ in the direction $k$ as shown in Fig. 4; $Q^{i i}(\mathrm{x}, t)$ is self-collision, representing the collision of particles of the species $i$; and $Q^{i j}(\mathrm{x}, t)$ is cross-collision, representing the collision between particles of species $i$ and of specie $j$.

In the absence of cross-collisions, Eq.1 reduces to the single-component, single-phase LB model presented in our previous work [25]. Since the molecular weights of the three species are different, their associated velocities $\xi_{k}^{i}$ are also different. At location $\boldsymbol{x}$ and at time $t$, the density $\rho_{i}$ and the velocity $\mathrm{u}_{i}$ of species $i$ are calculated respectively from; 


$$
\begin{aligned}
& \rho_{i}(x, t)=\sum_{k} f_{k}^{i}(x, t), \\
& \rho_{i} u_{i}(x, t)=\sum_{k} f_{k}^{i}(x, t) \xi_{k}^{i}
\end{aligned}
$$

The total mass density and average bulk velocity of the system is calculated from;

$$
\begin{aligned}
& \rho(x, t)=\sum_{j} \rho_{j}(x, t) \\
& \rho(x, t) \boldsymbol{u}(x, t)=\sum_{j} \rho_{j}(x, t) u_{j}(x, t)
\end{aligned}
$$

Following Luo et al. [29], the self-collision is described by the following single-relaxation time approximation;

$Q_{k}^{i i}(x, t)=\frac{1}{\tau_{i}}\left[f_{k}^{i[0]}(x, t)-f_{k}^{i}(x, t)\right]$

where $\tau_{i}$ is a dimensionless parameter, relating to the viscosity of species $i$. The cross-collision between particles of specie $i$ and specie $j$ is described by the following approximation;

$$
Q_{k}^{i j}(\boldsymbol{x}, t)=-\frac{1}{\tau_{i j}} \frac{\rho_{i}}{\rho} \frac{f_{k}^{i[e q]}}{\delta t \cdot c_{i}^{2}}\left(\xi_{k}^{i}-u\right)\left(u_{i}-u_{j}\right)
$$

Equation 5

where $c_{i}$ is the speed of sound of species $i$. The term $f_{k}^{i[0]}(\mathrm{x}, t)$ in Eq. 4 is the equilibrium distribution function. The values of $f_{k}^{i[0]}(\mathrm{x}, t)$ and $f_{k}^{i[e q]}(\mathrm{x}, t)$ are defined by; 


$$
\begin{aligned}
& f_{k}^{i[0]}(\boldsymbol{x}, t)=\left[1-\frac{\left(\boldsymbol{\xi}_{k}^{i}-\mathbf{u}\right)\left(\boldsymbol{u}_{i}-\boldsymbol{u}\right)}{c_{i}^{2}}\right] f_{k}^{i[e q]}(\boldsymbol{x}, t) \\
& f_{k}^{i[e q]}(\boldsymbol{x}, t)=w_{k} \rho_{i}\left[1+\frac{\boldsymbol{\xi}_{k}^{i} \cdot \boldsymbol{u}}{c_{i}^{2}}+\frac{\left(\boldsymbol{\xi}_{k}^{i} \cdot \boldsymbol{u}\right)^{2}}{2 c_{i}^{4}}+\frac{\boldsymbol{u} \cdot \boldsymbol{u}}{2 c_{i}^{2}}\right]
\end{aligned}
$$

respectively, where $w_{k}$ is a weight factor associated with $\boldsymbol{e}_{k}$ shown in Fig. 3 in that $w_{0}=3 / 9, w_{k}=1 / 18$ $(k=1-6)$ and $w_{k}=1 / 18(k=7-18)$.

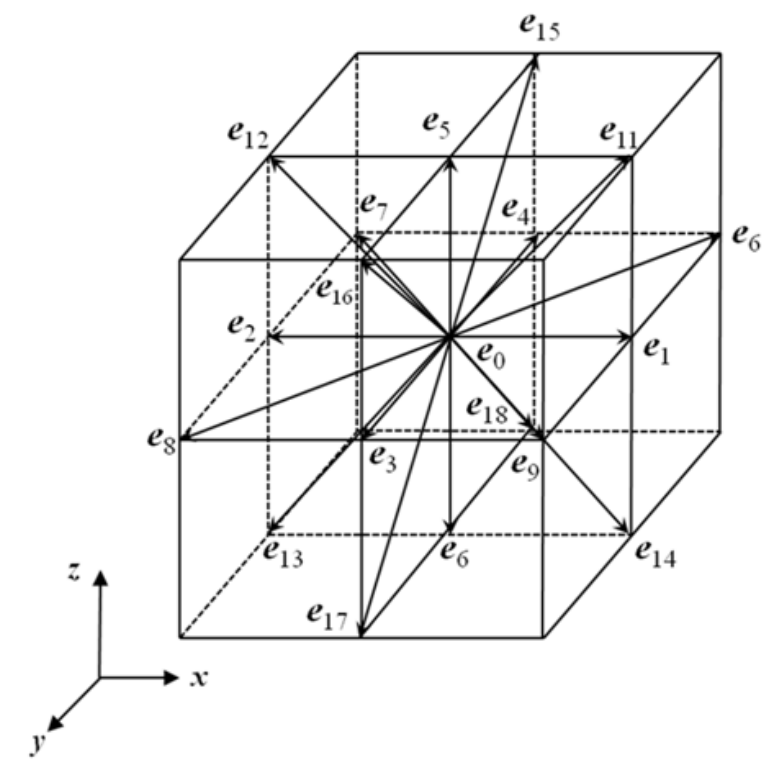

Figure 4 The nineteen velocities in the three dimensional modelling domain (D3Q19) for the developed single-phase multi-component lattice Boltzmann numerical model

\section{Simulating Particle Movement in a Three-Species System}

For fluid transport in the GDL, it is sufficient to consider three species $(i, j=1,2,3)$ for both cathode and anode. If species 1 is assumed to the lightest fluid with a molecular weight of $M_{1}$, specie 3 the heaviest with a molecular weight of $M_{3}$, and the molecular weight of species $2 M_{2}$ which lies 
between them, the velocities along which the particles of specie 1 move are $\boldsymbol{\xi}_{k}^{1}=\boldsymbol{e}_{k}$, that is, $\boldsymbol{\xi}_{0}^{1}=(0,0,0), \quad \xi_{k}^{1}=( \pm \delta x / \delta t, 0,0) \quad$ for $k=1,3 ; \quad \xi_{k}^{1}=(0, \pm \delta x / \delta t, 0) \quad$ for $k=2,4 ; \quad \xi_{k}^{1}=(0,0, \pm \delta x / \delta t)$ for $k=5,6 ; \quad \xi_{k}^{1}=( \pm \delta x / \delta t, \pm \delta x / \delta t, 0) \quad$ for $\quad k=7 \sim 10 ; \quad \xi_{k}^{1}=( \pm \delta x / \delta t, 0, \pm \delta x / \delta t) \quad$ for $\quad k=11 \sim 14 ;$ $\xi_{k}^{1}=( \pm \delta x / \delta t, 0, \pm \delta x / \delta t)$ for $k=15 \sim 18$; where $\delta x$ is the side length of the voxles of the X-ray images and $\delta t$ is time step. The respective velocities of the particles of specie 2 are $\xi_{k}^{2}=\sqrt{M_{1} / M_{2}} \xi_{k}^{1}$, and of specie 3 are $\xi_{k}^{3}=\sqrt{M_{1} / M_{3}} \xi_{k}^{1}$.

In the above the LB model, the kinematic viscosity of species $i(i=1,2,3)$ is determined by the self-collision relaxation parameter in $\mu_{i}=\left(\tau_{i}-0.5\right) \delta x^{2} / \delta t \sqrt{M_{1} / M_{i}}$ and the partial pressure by $p^{i}=\rho^{i} \delta x^{2} /\left(3 \delta t^{2}\right) \sqrt{M_{1} / M_{i}}$. The binary diffusion coefficient of species pair $i$ and $j$ is given by:

$D_{i j}=\frac{\rho P}{b^{2} M_{i} M_{j}}\left(\tau_{i j}-0.5\right)$

Equation 8

where $P=\sum_{i} p^{\sigma}$ is the total pressure and $n=\sum_{i} \rho^{i} / M_{i}$.

\section{Computational Steps}

For each species, the implementation of the above LB models involves two steps to advance one computational time step. The first is the collision step, the purpose of which is to calculate the term on the right-hand side of Eq. 1 as $f_{k}^{i[*]}(x, t)=f_{k}^{i}(x, t)+Q_{k}^{i i}(x, t)+\sum_{i \neq j} Q_{k}^{i j}(x, t)$. The second is the streaming step and its purpose is to advance the result of the collision to the new position to become $f_{k}^{i}\left(\mathrm{x}+\xi_{k}^{i} \delta t, t+\delta t\right)=f_{k}^{i[*]}(\mathrm{x}, t)$ after a time period of $\delta t$. For all the three species, the particles distribution functions are defined at the centres of each volume element in the 3D model, i.e., a voxel. 
From the definitions of the particle velocities of each species, it is therefore known that after streaming, the particles of specie 1 will move from the centre of one voxel exactly to the centres of its adjacent voxels within one time step. The particle velocities for specie 2 and 3 in the meantime are slower. As a result, during the period of one time step, these particles can only move to somewhere between the centre of the voxel where the particles originate and the centres of its neighbouring voxels. In this paper, first-order and second-order interpolations have been employed to estimate the particle distributions of species 2 and 3 at the centre of a voxel.

A final issue to clarify is the treatment of the solid surface when streaming the particles from one place to another. In this paper, the solid-void interface is treated as an impermeable and non-slip boundary where the bulk velocities of all the three species are zero; the non-slip boundary has been solved by using the bounce-back method, as described in our previous work [25].

\section{Interfacial Conditions}

The GDL-channel interface is an inlet boundary for the LB model from which all the species emanate for the LB model. The partial pressure of each species is specified at this boundary and it is assumed that these do not change with time. Such boundary was treated by the method proposed in our previous work [30] The GDL-catalyst interface is an outlet boundary, and its impact on gas flow varies from species to species. The consumption rate for oxygen (cathode GDL) is known at the outlet boundary from the current density. Since the PEM is assumed to be impermeable to gases for the purposes of this study, the flow rate of inert gases are set to zero at the GDL-catalyst layer interface. For nitrogen therefore, this boundary was treated as a barrier and solved for by the bounce-back method, as discussed above. 
Due to the cross-flow of water across the cell via the PEM it has been assumed that water vapour can move through the catalyst layer into the membrane. Therefore, in a similar manner as for hydrogen and oxygen, the flux rate of water vapour at the outlet boundary is specified through the electrochemical model based on the current density and the determined net water flux ratio. The flow rate of each species includes convection and diffusions. In the multiple-component LB model the flow rate of the species $i$ is calculated from the particle distribution functions of all three species as follows [29]:

$\boldsymbol{q}_{i}=\sum_{k=1}^{18} f_{k}^{i} \boldsymbol{\xi}_{k}^{i}+\sum_{j \neq i} \frac{1}{2 \rho \tau_{i j}}\left(\rho_{i} \sum_{k=1}^{18} f_{k}^{j} \xi_{k}^{j}-\rho_{j} \sum_{k=1}^{18} f_{k}^{i} \xi_{k}^{i}\right)$

Equation 9

Equation 9 is used to treat the water vapour and oxygen at the outlet boundary where their flow rate are specified. The unknown variables on the outlet boundaries are calculated through extrapolation.

\subsection{Electrochemical Model}

\section{The General Transport Equation}

The one-dimensional electrochemical model employed for the current study is based on the General Transport Equation (GTE), which is derived from fundamental molecular theory [3,4]. In general, the GTE describes the movement of a species as part of a multi-component concentrated solution system due to the following modes of transport:

- diffusion due to concentration gradients

- convection due to pressure gradients

- thermal diffusion due to temperature gradients 
- $\quad$ electro-osmotic drag due to an electric field

In its generalised form, the GTE appears as follows for a species $i$;

$$
\begin{aligned}
c_{i}\left[\nabla \mu_{i}+\bar{s}_{i} \nabla T-\frac{M_{i}}{\rho} \nabla p\right]- & R T \dot{n}_{H+} \frac{c_{m}}{C_{T}}\left(\frac{\xi_{i}}{D_{i, m}}-\frac{\rho_{i}}{\rho} \sum_{j \neq i, m e m}^{v} \frac{\xi_{j}}{D_{j, m}}\right) \\
= & R T \sum_{j} \frac{c_{i} C_{j}}{c_{T} D_{i j}}\left[v_{j}-v_{i}+\left(\frac{D_{j}^{T}}{\rho_{j}}-\frac{D_{i}^{T}}{\rho_{i}}\right) \nabla \ln T\right]
\end{aligned}
$$

where $c_{i}$ is the molar concentration, $\mu_{i}$ is the electrochemical potential, $\bar{s}_{i}$ is the molar entropy, $\dot{n}_{i}$ is the molar flux rate, $\xi_{i}$ is the electro-osmotic drag ratio, $v_{i}$ is the molecular velocity and $D_{i}^{T}$ is the thermal diffusion coefficient. For the purposes of PEFC modelling in the current study, it is assumed that the temperature gradients generated within the material of a PEFC in the principal through-plane flow direction do not invoke thermal diffusion.

\section{Mechanisms of Electrochemical Transport in Porous and Quasi-Porous Systems}

The premise of the electrochemical model is that the GTE can be applied to simulate electrochemical transport through both porous and quasi-porous materials in the PEFC. While Eq. 10 can be applied directly with the assumption that $\nabla T=0$ to simulate transport through porous layers, it is modified to the following form to simulate transport through quasi-porous layers, accommodating potential liquid-induced expansion of internal pores [4];

$\nabla c_{w}=\frac{1}{c_{\text {tot }}}\left(s_{e p f}\left(I \Phi_{2, \text { liq }}+\Phi_{3, \text { liq }}\right)+\left(1-s_{\text {epf }}\right)\left(I \Phi_{2, \text { vap }}+\Phi_{3, \text { vap }}\right)-I \Phi_{1} \alpha\right)$

where 


$$
\begin{aligned}
& \Phi_{1}=\left[\frac{c_{H+}}{D_{w, H+}}+\frac{c_{m e m}}{D_{w, m e m}}\right] ; \\
& \Phi_{2, J}=\frac{c_{w}}{D_{w, H+}}+\frac{c_{m} \xi_{w, J}}{D_{w, m e m}} ; \\
& \Phi_{3, J}=\frac{c_{t o t} \rho_{w}}{R T \rho} \nabla P_{J}
\end{aligned}
$$

where $I$ is the molar flux of water generated by the cell in the cathode CL due to the cell current and $s_{\text {epf }}$ is the fraction of pores in the quasi-porous polymer electrolyte which are forcibly expanded by the infiltration of liquid water.

Pressure gradients for a species $J$ through porous and quasi-porous layers are calculated through Darcy's law:

$$
\nabla P_{J}=-\frac{\dot{n}_{J} \mu_{J}}{c_{J} k_{J} K}
$$

where $\mu_{J}$ is phase viscosity, $k_{J}$ is the permeability pre-factor which accounts for liquid saturation and $K$ is the absolute permeability.

\section{Modelling Structure}

The electrochemical model contains three repeatable sub-models which represent elements in the physical system. The first sub-model is for the anode and cathode channels. It determines the conditions in both reactant supply channels based on a zero-dimensional treatment by accounting for the conditions of both inlet gases, the consumption of reactants due to the current density, the generation of product 
water and the cross-flow through the cell. The second sub-model is for the anode and cathode porous layers, and based principally on Eqs. 10 and 13. Based on the initial value boundary conditions in each of the gas supply channels from the channel model, the porous-layer sub-model determines the distribution of gas components and the infiltration of water through the anodic and cathodic porous layers using a one-dimensional treatment. The third and final sub-model is for the quasi-porous PEM and based principally on Eqs. 11 - 13. The quasi-porous layer sub-model uses the boundary conditions at the interface between the PEM and the anode GDL as initial values to determine the conditions at the interface between the PEM and the cathode GDL (interface C2 in Fig. 2). A critical parameter - the net water flux ratio - is iteratively determined ensuring that the conditions at C2 generated from opposing directions are identical. A full description of the mechanistic treatment is beyond the scope of the current study and provided elsewhere [4]. The general modelling scheme is described in Fig. 5.

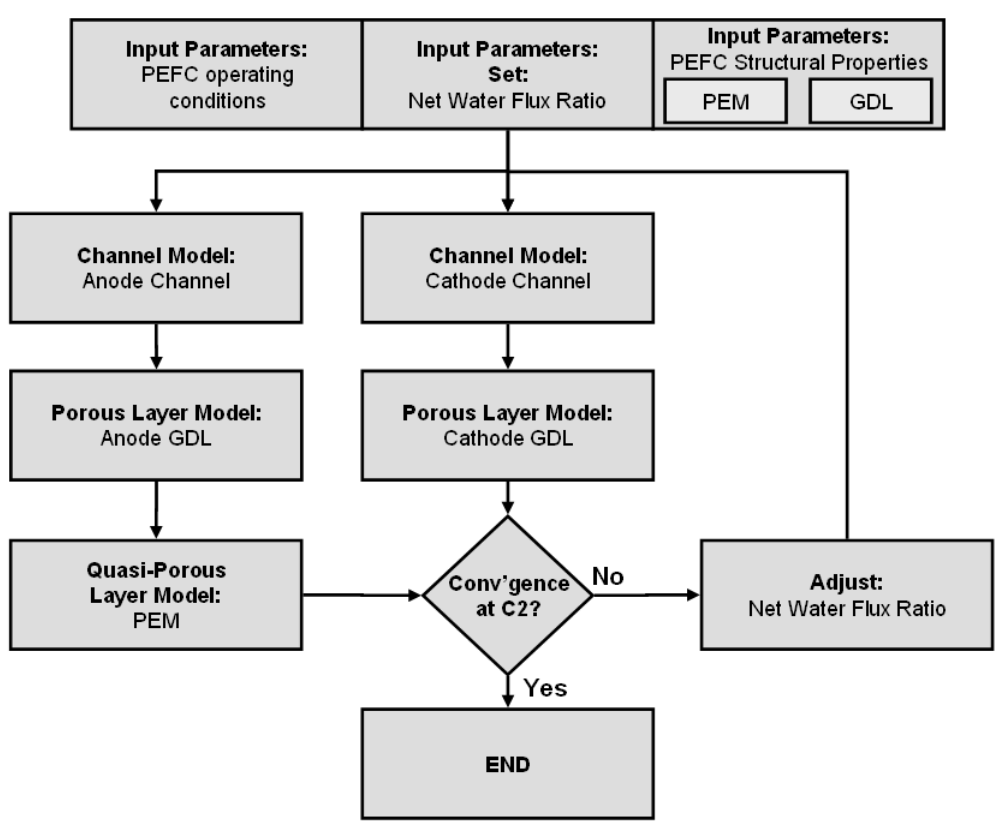

Figure 5 General Modelling Scheme of the Electrochemical Model based on the GTE

\subsection{X-Ray Micro-Tomography}


A full description of the X-ray tomography procedure is provided elsewhere but discussed here in brief [31,32]. There are three basic steps in the procedure; progressive image acquisition, image processing and digital reconstruction. The aim of the first step is to obtain and compile a set of twodimensional X-ray micro-tomography images of the three-dimensional porous structure. The aim of the second step is to process the images in order to distinguish the difference between solid space and void spaces in the porous structure. The aim of the third step is to apply the determined threshold level in order to regenerate a digital model of the three-dimensional porous structure in binary format where 1 represents solid space and 0 represents pore space.

\section{Image Acquisition via $X$-ray Micro-Tomography}

The two-dimensional X-ray shadow images of the GDL sample are obtained by placing the GDL sample in front of an X-ray source and progressively rotating the sample by 0.9 degrees. In the technique developed here to characterise PEFC materials, the sample does not need any special pretreatment. Images are generated in the current study using a SkyScan 1072 X-ray micro-tomography system, which has an X-ray source of $50 \mathrm{kV}$ at $100 \mu \mathrm{A}$. The image is rotated through a total of 180 degrees, resulting in 200 shadow images. The image acquisition process requires 45 minutes.

The image acquisition system contains a Hamamatsu X-ray camera for X-ray detection which contains a scintillator and a charge coupled device (CCD) chip. The godalinum oxide scintillator screen coverts the X-rays into flashes of light. Potential damage to the CCD chip due to prolonged exposure to X-rays is prevented through the use of a tapered fibre-optic bundle, which is glued to the scintillator screen at one end and the CCD chip at the other. The fibre-optic bundle maintains an exact 1:1 translation. The CCD chip in the SkyScan 1072 system has a $1024 \times 1024$ pixel resolution with a 12-bit 
depth. The exact object diameter is $1.78 \mathrm{~mm}$ and therefore the maximum resolution of the system is 1.74 $\mu \mathrm{m}$.

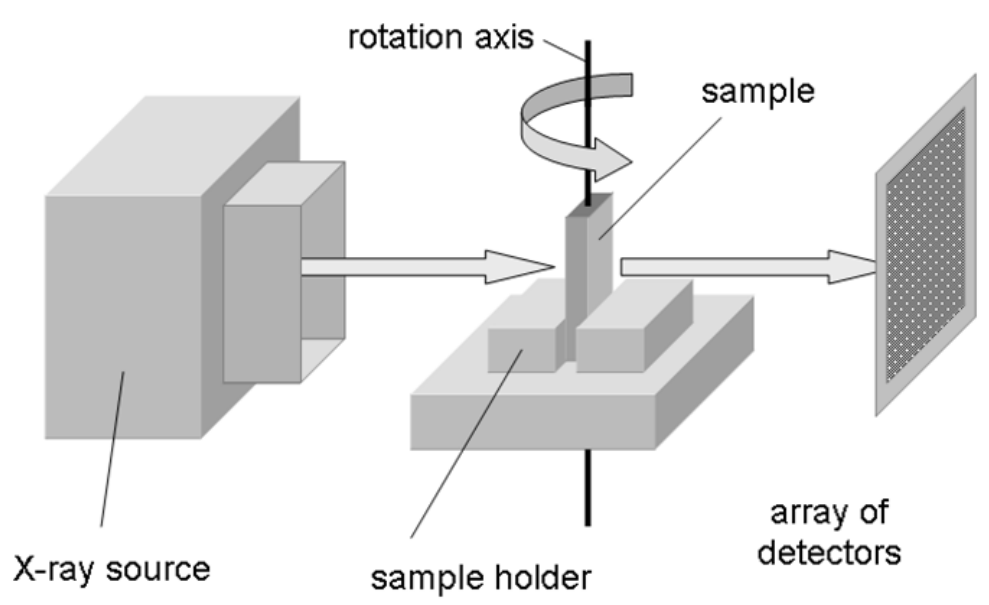

Figure 6 Principle of the micro-tomography system

\section{Image Processing via Heuristic Thresholding}

The 2D greyscale images generated using X-ray tomography system during the image acquisition process are subsequently compiled using the program CTAN [33] to generate 2D crosssectional image slices in a greyscale format (256 shades of grey). These images correspond to what would be seen if the sample is cut through the scanning plane. A further process has to be applied in order to generate a digital 3D model for LB simulations; this is carried out using a thresholding process.

The thresholding process defines a partition on the greyscale where darker regions of the 2D image slices correspond to solid space while lighter regions correspond to void space. In order to determine the appropriate threshold level, a heuristic technique is employed whereby a comparison is made between the geometric features of a 2D image of the GDL surface which is obtained using scanning electron microscopy (SEM), and those of the thresholded 2D greyscale images which are generated using CTAN. The SEM image is obtained at a resolution of $400 \mathrm{~nm}$ and treated as a reference 
image. The average fibre diameter in the SEM image can be calculated as the total area of the fibres divided by the total length of the fibres using CTAN software. The average fibre diameter determined from the reference image is assumed to be representative of that for the entire porous 3D structure. A common threshold level is then applied to the $2 \mathrm{D}$ image slices and progressively tuned to meet two objectives:

(a) the average fibre diameter of the $2 \mathrm{D}$ image slices must match that measured in the reference image, and;

(b) by visual inspection, the fibre continuity on the surface of the stack of 2D image slices model must be preserved relative to the reference image.

The correct threshold level is deemed to have been achieved when both of the above objectives are satisfied.

\section{Reconstruction of 3D Digital Model}

The final step involves generating a three-dimensional digital model of the porous material using the thresholded greyscale 2D image slices generated during the image processing step. There are several algorithms that can be employed to carry this out, for example Double Time Cubes and Matching Cubes [30]. The resulting digital 3D model can subsequently be used directly with the LB model without modification in order to simulate flow through its porous structure.

\section{$5 \quad$ Results and Discussions}

\subsection{Three-dimensional Digital Model of the GDL}


In the current work, the average fibre diameter measured from the SEM reference image is 7.5 $\mu \mathrm{m}$, which compares to $7.9 \mu \mathrm{m}$ from the same surface of the $3 \mathrm{D}$ digital model; the error in the average fibre diameter as a result of threshold tuning is therefore 5\%. Figure 7 shows an image of a region of the GDL imaged and that proportion which is used for the SPMC-LB simulation. The digital model for SPMC simulation has a pixel size of 45 by 45 in the $x-y$ plane and 145 pixels along the z-plane, which is parallel to the principal flow direction. Given that the resolution of the X-ray tomography system is 1.74 $\mu \mathrm{m}$, the size of the 3D image is 78.3 by 78.3 by $252.3 \mu \mathrm{m}^{3}$.

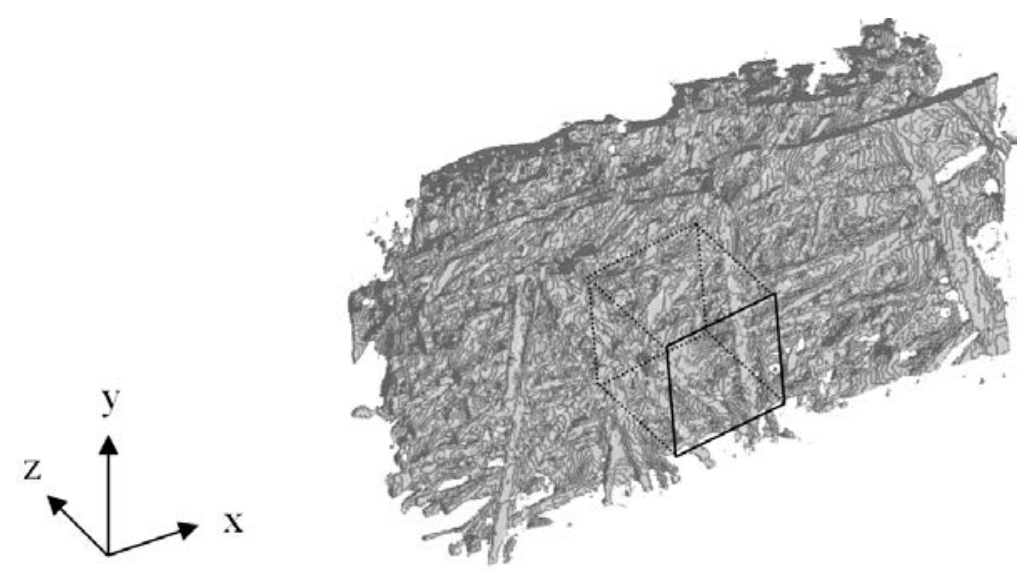

Figure 7 X-ray tomography image of carbon paper GDL with 3D region of interest shown. Region of interest is $78.3 \times 78.3 \times 252.3 \mu \mathrm{m}^{3}$.

By applying the SPSC LB model to the digital image shown in Fig. 7, it is possible to reveal rudimentary characteristic properties of its porous structure such as porosity and tortuosity. This can then be supplied to the electrochemical model. Accordingly, the SPSC model reveals that the porosity of the reconstructed digital model is 0.84 . The tortuosity in the principal through-plane flow direction is calculated to be 1.15. Fig. 8 details the 3D stream tubes along which infiltrating gases are transported. The solid region shown in Fig. 7 is removed from the image given in Fig. 8. The stream tubes reveal that the heterogeneity of the porous structure forces flow to coalesce along dominant, tortuous flow paths. 


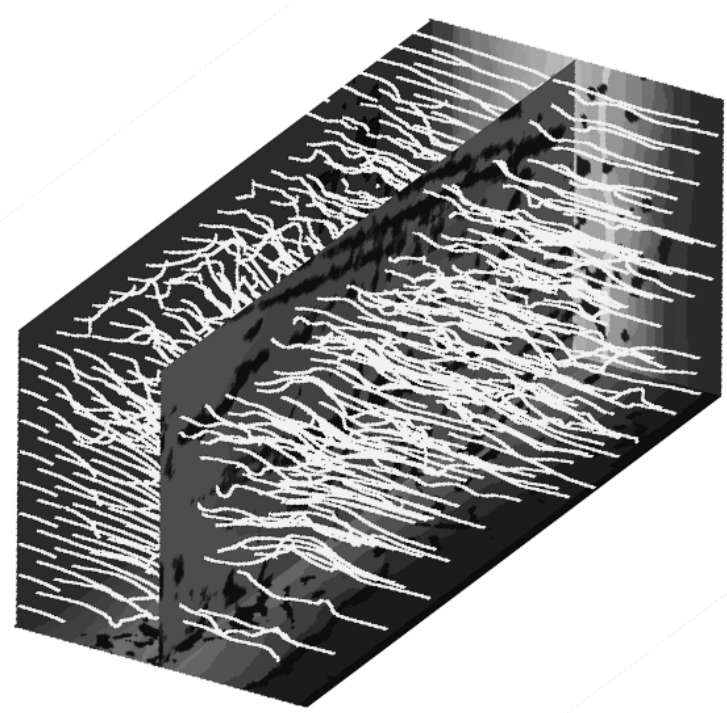

Figure 8 Simulated 3D stream tubes for the reconstructed digital image of the carbon paper GDL shown in Figure 7

\subsection{Boundary Conditions and Flow Rates from the Electrochemical Model}

To cross-validate the electrochemical and lattice Boltzmann models, four test conditions were generated, which covered a range of humidified conditions for the PEFC. These are summarised in Tab. 1. The physical properties of the fuel cell layers and the thermodynamic operating conditions applied in the simulation are given in Tab. 3. The simulations were run at $0.5 \mathrm{~A} / \mathrm{cm}^{2}$ current density. Figure 9a shows the boundary pressures at interface C1, as predicted by the electrochemical model. Figure 9b shows the calculated flow rates of water and oxygen through the cathode gas diffusion layer, again as predicted by the electrochemical model.

\begin{tabular}{lll} 
& Anode & Cathode \\
\hline Case 1: $\boldsymbol{A D C D}$ & Dry & Dry \\
Case 2: $\boldsymbol{A D C W}$ & Dry & Wet $\left(100 \% \mathrm{RH}\right.$ at $\left.70^{\circ} \mathrm{C}\right)$ \\
Case 3: $\boldsymbol{A W C D}$ & Wet $\left(100 \% \mathrm{RH}\right.$ at $\left.70^{\circ} \mathrm{C}\right)$ & Dry \\
Case 4: $\boldsymbol{A W C W}$ & Wet $\left(100 \% \mathrm{RH}\right.$ at $\left.70^{\circ} \mathrm{C}\right)$ & Wet $\left(100 \% \mathrm{RH}\right.$ at $\left.70^{\circ} \mathrm{C}\right)$
\end{tabular}

Table 1 Test Cases for model-to-model validation 


\begin{tabular}{ll} 
Property & Value \\
\hline GDL porosity & $84 \%$ \\
GDL tortuosity & 1.14 \\
GDL thickness, $\mu \mathrm{m}$ & 252.30 \\
PEM thickness, $\mu \mathrm{m}$ & 25.40 \\
PEM equivalent weight, g/cm ${ }^{3}$ & 1100 \\
PEM dry density, g/cm ${ }^{3}$ & 2.00 \\
Anode pressure, bar & 1.50 \\
Cathode pressure, bar & 1.00 \\
Dry cathode gas composition & $0.21 / 0.79 \mathrm{O}_{2} / \mathrm{N}_{2}$ \\
Dry anode gas composition & $1.00 \mathrm{H}_{2}$ \\
Anode dry gas flow rate, slpm & 0.20 \\
Cathode dry gas flow rate, slpm & 0.90 \\
Cell temperature, ${ }^{\circ} \mathrm{C}$ & 70.00 \\
Cell area, cm ${ }^{2}$ & 5.00
\end{tabular}

Table 2 Physical properties of fuel cell layers and thermodynamic operating conditions for electrochemical model simulations

The results in Fig. 9a shows that the partial pressure of water vapour at interface C1 increases when the cathode is humidified, compared to when it is supplied dry. For the ADCW and AWCW test cases, this has the effect of reducing the nitrogen partial pressure by $29 \%$ and $32 \%$ respectively compared to the ADCD case. The oxygen partial pressure falls as well by $28 \%$ and $31 \%$ respectively. The results in Fig. $9 \mathrm{~b}$ show that when both gases are supplied dry, water vapour flows out of the cathode GDL into the channels. If the cathode gas is then humidified, water flows in to the cathode GDL. If the anode gas is humidified instead, water vapour reverts to flowing out of the cathode GDL. With both gases fully humidified, water vapour continues to flow out of the cathode. The results therefore suggest that without cathode humidification, the cathodic PEM boundary is insufficiently hydrated to allow molecular diffusion to overcome electro-osmotic drag; water vapour therefore leaves the cathode GDL. Even in the case where both gases are humidified, it is the electro-osmotic drag which prevails. If the anode gas is dry while the cathode gas is humidified, the diffusive flux prevails and therefore water vapour flows into 
the cathode GDL. Oxygen always flows into the cathode GDL due to the electro-reduction process in the cathode catalyst layer.

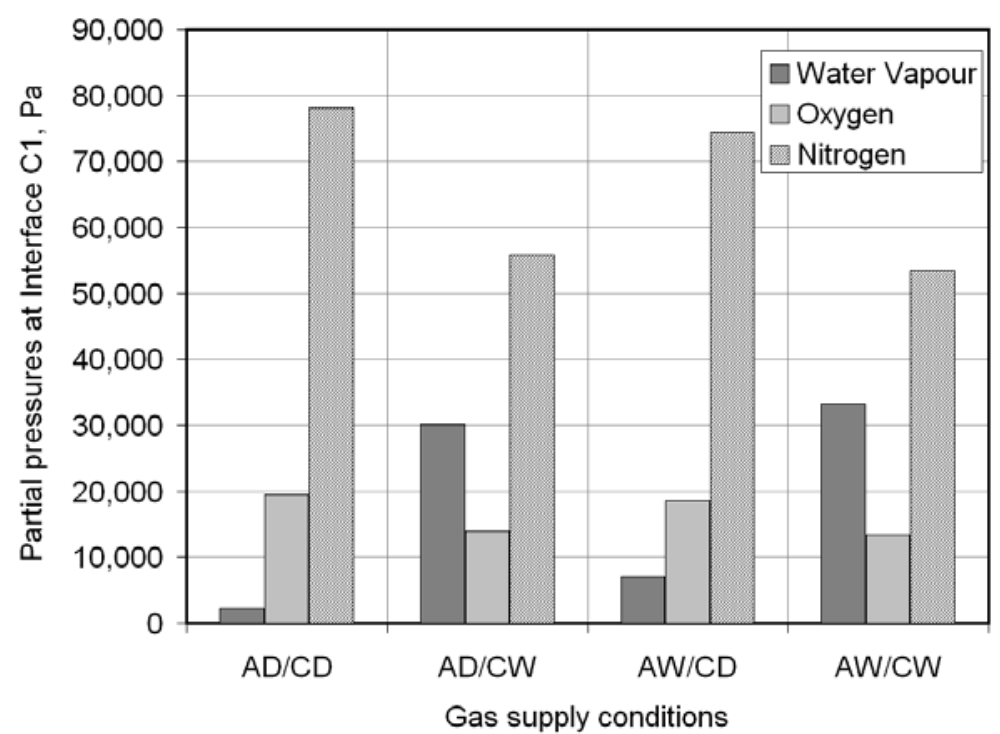

(a)

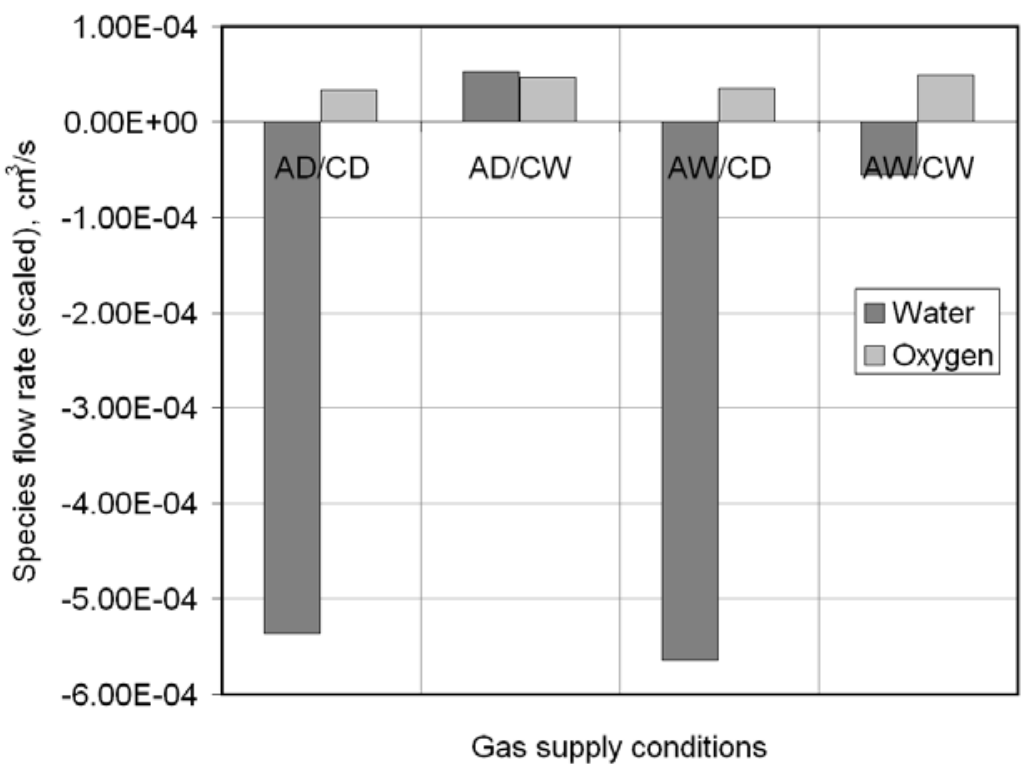

(b)

Figure 9. Simulated boundary conditions from the electrochemical model for the lattice Boltzmann model (a) simulated partial pressures at interface C1 (b) simulated species flux data 


\subsection{Simulated multi-species distribution through the cathode GDL through lattice Boltzmann modelling}

The partial pressures supplied in Fig. 9a and the flow rates in Fig. 9b are supplied directly to the lattice Boltzmann model in order to simulate the distribution of cathode gases through a proportion of the digital 3D model of the carbon paper GDL as shown in Fig. 7. Figures 10 a-d shows the resulting partial pressure distribution of oxygen through a 78.3 by 78.3 by $252.3 \mu \mathrm{m}^{3}$ volume of the 3D heterogeneous structure for the four test conditions described in Tab. 1. The 3D distributions can be averaged in the $x-y$ plane along the z-axis in order to generate a 1D profile (i.e., parallel to the principal flow direction). This curve can then be compared to the macroscopic 1D distributions generated by the electrochemical model.
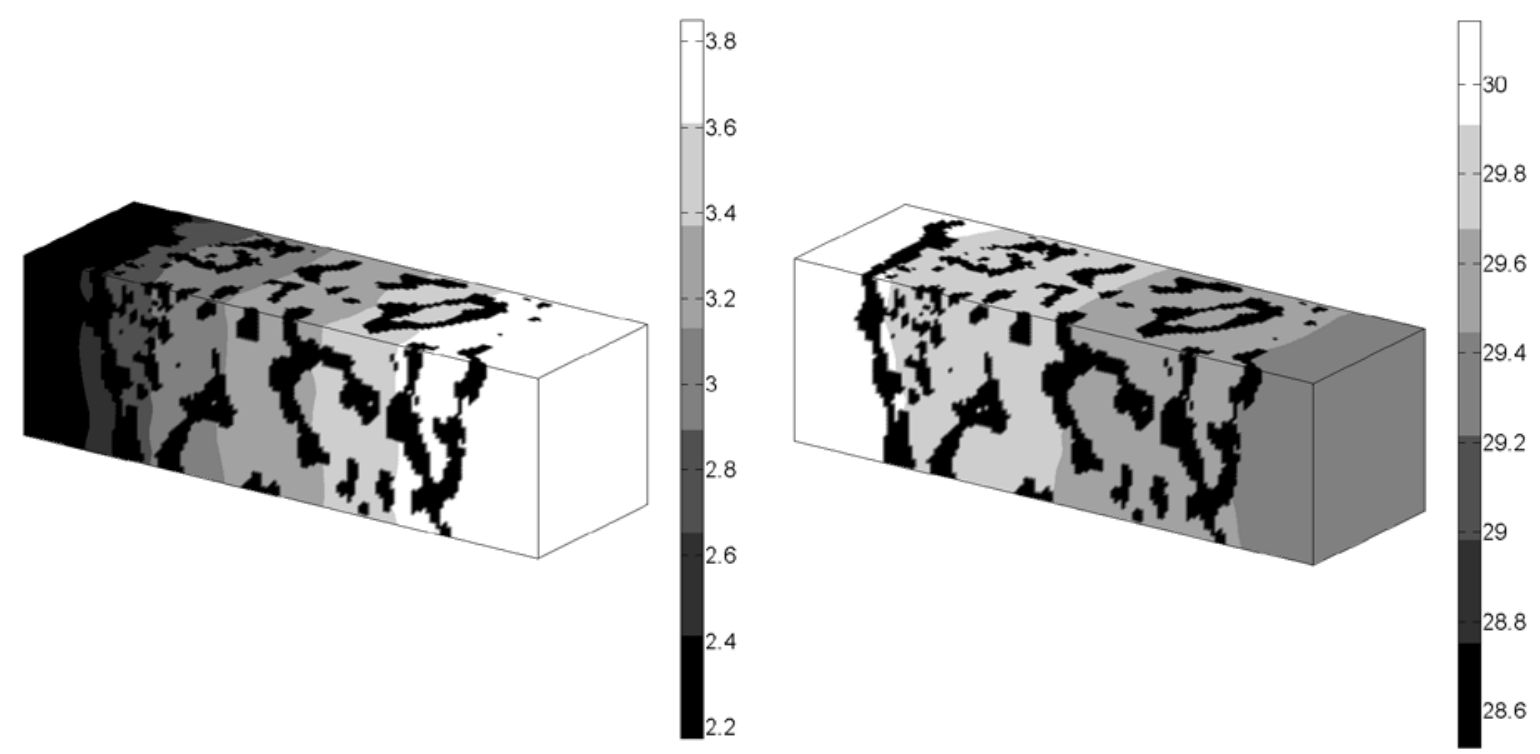

(a)

(b) 


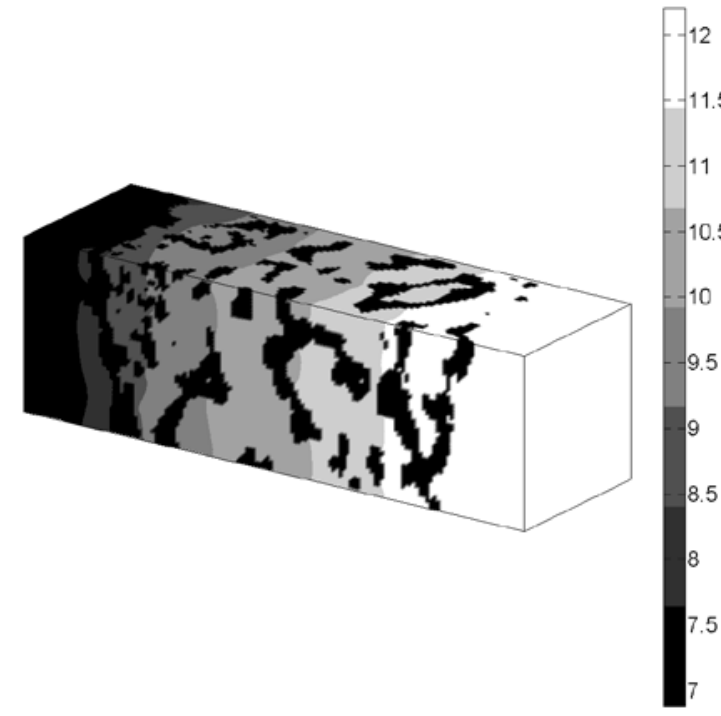

(c)

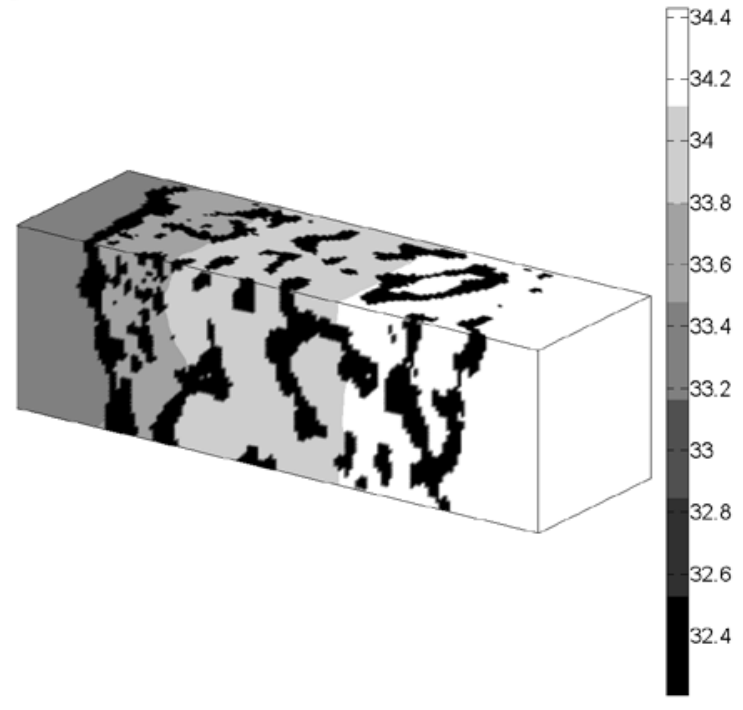

(d)

Figure 10. SPMC-LB simulation of oxygen distribution through a 78.3 by 78.3 by 252.3 $\mu \mathrm{m}^{3}$ volume of the carbon paper GDL digital model; (a) ADCD, (b) ADCW; (c) AWCD; (d) AWCW. All pressures are shown in $\mathrm{kPa}$

Figures 11 a-c compare the gas distribution profiles for water, oxygen and nitrogen simulated by the LB model to that calculated by the electrochemical model for test case 1 . Both sets of results suggest that the water pressure increases from the channel/GDL interface towards the catalyst layer/GDL interface, which is opposite in direction to the flux of water. The LB treatment suggests a total pressure rise of $1.61 \mathrm{kPa}$ from interface $\mathrm{C} 1$ to $\mathrm{C} 2$, whereas the macroscopic treatment suggests $0.81 \mathrm{kPa}$. As oxygen travels towards the catalyst layer from the channel boundary, its pressure drops throughout the thickness of the GDL, as shown by both curves in Fig. 11b. The LB treatment suggests a pressure drop of $1.06 \mathrm{kPa}$ whereas the macroscopic treatment suggests $0.71 \mathrm{kPa}$. Both simulations also suggest that the nitrogen partial pressure drops towards the catalyst layer, by $1.31 \mathrm{kPa}$ according to the LB model but only $0.1 \mathrm{kPa}$ according to the macroscopic model. 


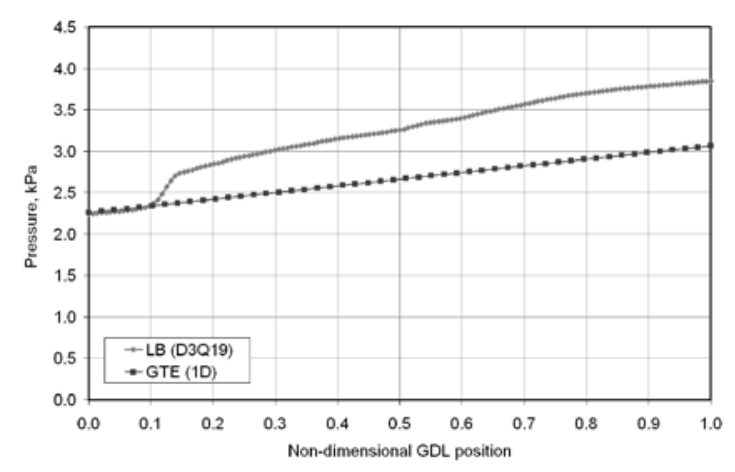

(a)

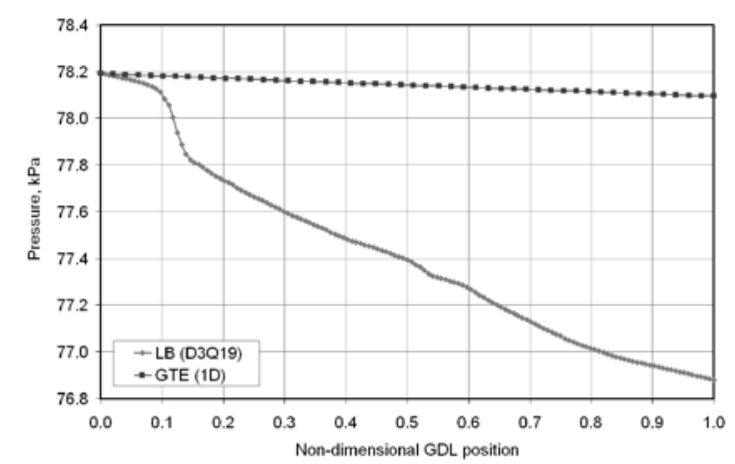

(c)

Figure 11. Comparison of lattice Boltzmann and macro-homogeneous predictions of gas pressure profiles along the GDL: anode dry and cathode dry cell operating conditions; (a) water vapour; (b) oxygen; (c) nitrogen

Figures 12 a-c shows the pressure profiles for test case 2.As shown in Figure 9b, under these test conditions water enters the cathode GDL from the channel, travelling in the direction of the catalyst layer interface. As such, the pressure gradient for water vapour is negative, as shown in Fig. 12a. The LB method suggests that the pressure drop is $0.74 \mathrm{kPa}$ and the macroscopic treatment suggests a similar value of $0.61 \mathrm{kPa}$. Oxygen reduction causes water to travel towards the catalyst layer interface once again, resulting in a pressure drop. Again, the superficial pressure drops predicted by the two methods are similar, being $0.47 \mathrm{kPa}$ for the LB method and $0.37 \mathrm{kPa}$ for the macroscopic method. The partial 
pressure of nitrogen increases through the cathode GDL in the direction of the cathode catalyst layer, compensating for the pressure drop of water vapour and oxygen.
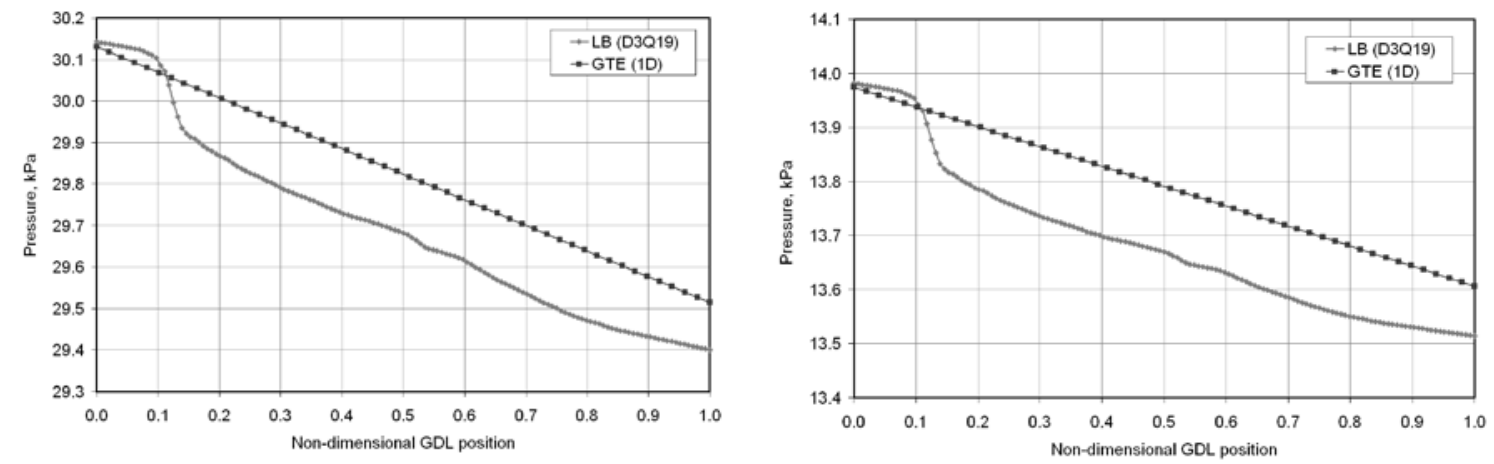

(a)

(b)

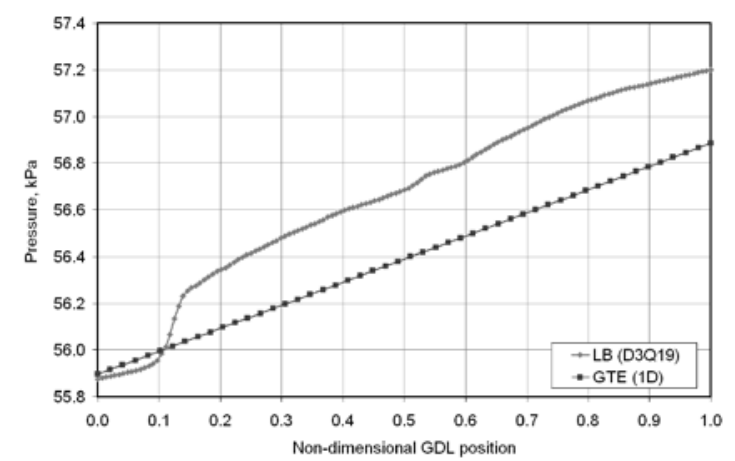

(c)

Figure 12. Comparison of lattice Boltzmann and macro-homogeneous predictions of gas pressure profiles along the GDL: anode dry and cathode wet cell operating conditions; (a) water vapour; (b) oxygen; (c) nitrogen

For test case 3, the wet anode and dry cathode inlet gases cause water to travel through the cathode GDL towards the channel. This results in a pressure rise in the reverse direction through the cathode GDL from the channel interface to the catalyst layer interface. This is shown in Fig. 13a. The LB calculation suggests a pressure rise of $5.10 \mathrm{kPa}$, while the macroscopic treatment calculates $2.5 \mathrm{kPa}$. The oxygen partial pressure continues to fall towards the cathode catalyst layer interface due to the 
reduction reaction, as shown in figure $13 \mathrm{~b}$. The pressure drop simulated by the LB model is $1.97 \mathrm{kPa}$ and $1.06 \mathrm{kPa}$ for the macroscopic treatment. The nitrogen partial pressure also falls by $5.29 \mathrm{kPa}$ according to the LB model and $1.48 \mathrm{kPa}$ according to the macroscopic model, as shown in Fig. 13c.
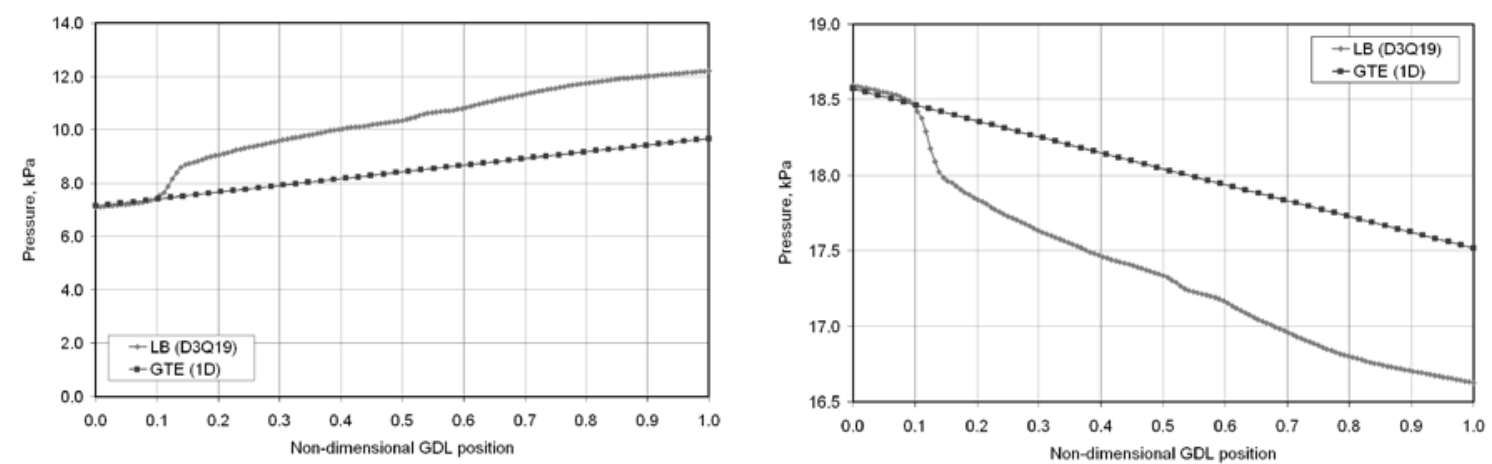

(a)

(b)

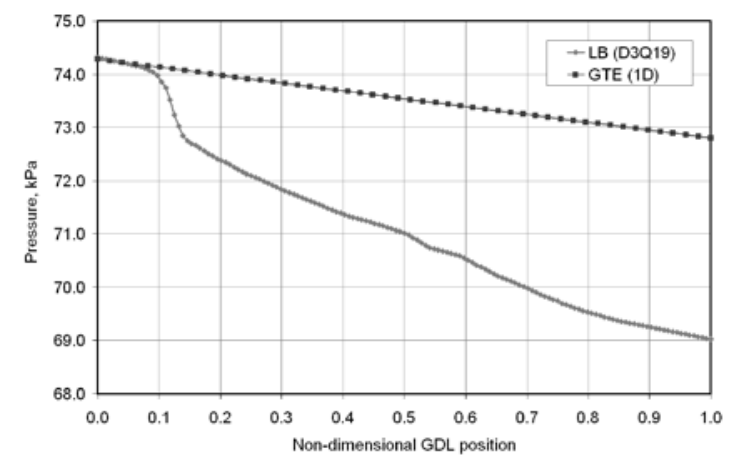

(c)

Figure 13. Comparison of lattice Boltzmann and macro-homogeneous predictions of gas pressure profiles along the GDL: anode wet and cathode dry cell operating conditions; (a) water vapour; (b) oxygen; (c) nitrogen

Figure 14 compares the results for the case where both anode and cathode gases are supplied fully humidified. The results suggest here that water vapour exits the cathode GDL and enters the cathode channel. Oxygen and nitrogen profiles exhibit similar a similar behaviour to that observed for test case 3 in Figs. 13b and 13c. The LB method suggests that the partial pressure of water rises towards 
the catalyst layer interface by $1.22 \mathrm{kPa}$. The oxygen partial pressure falls simultaneously by $0.96 \mathrm{kPa}$ while the nitrogen partial pressure falls by $0.83 \mathrm{kPa}$.
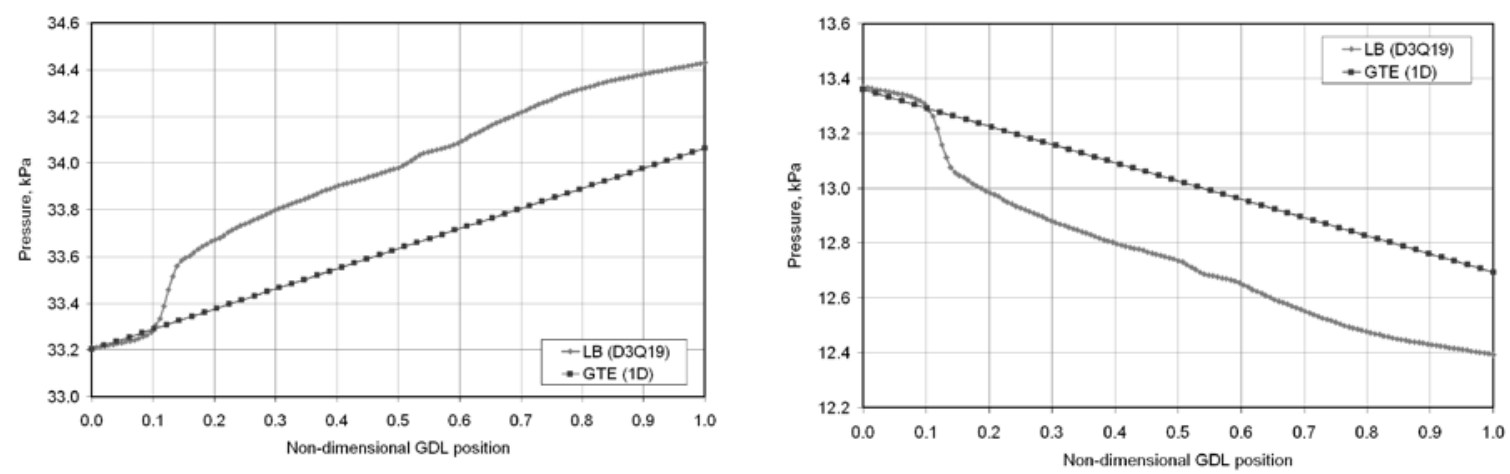

(a)

(b)

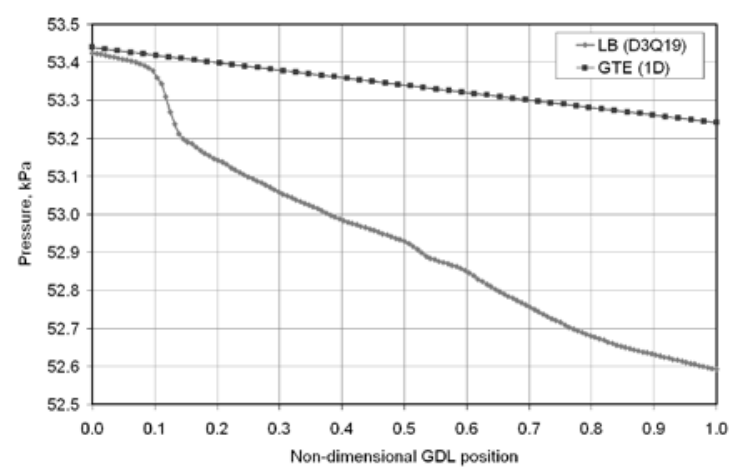

(c)

Figure 14. Comparison of lattice Boltzmann and macro-homogeneous predictions of gas pressure profiles along the GDL: anode wet and cathode wet cell operating conditions; (a) water vapour; (b) oxygen; (c) nitrogen

Figure 15 shows the 2D porosity of the digital model calculated for each $x$ - $y$ plane perpendicular to the principal flow direction (parallel to the z-axis). The graph shows that for the section of the digital model shown in Fig. 7 that is used for LB simulations, the outer extremities of the digital model are mainly characterised by void spaces ( $<5 \%$ of the thickness of the GDL closest to interface C1 and $<10 \%$ closest to interface C2). For the region closest to the interface C1 (non-dimensional GDL position of 
0.0), the high initial porosity drops to below $40 \%$ within the first $15 \%$ of the thickness of its thickness. The interference caused by the solid structure results in the abrupt pressure drop seen for all gases travelling towards interface C2 in figures $11-14$. Thereafter, the heterogeneous porous structure causes the permeability to fluctuate mainly between $65 \%$ and $90 \%$, rising to above $90 \%$ in the last $10 \%$ of the thickness closest to interface C2. Overall, therefore, Fig. 15 confirms that the heterogenic nature the carbon paper GDL can clearly influence the non-linearity of the pressure distribution of each species infiltrating its porous structure.

It is also acknowledged that excess void space on the outer extremities of the digital image can affect permeability, porosity, tortuosity and two-phase LB calculations and ideally should be eliminated prior to simulation, therefore. However, this is left at the present moment as a refinement exercise for future work.

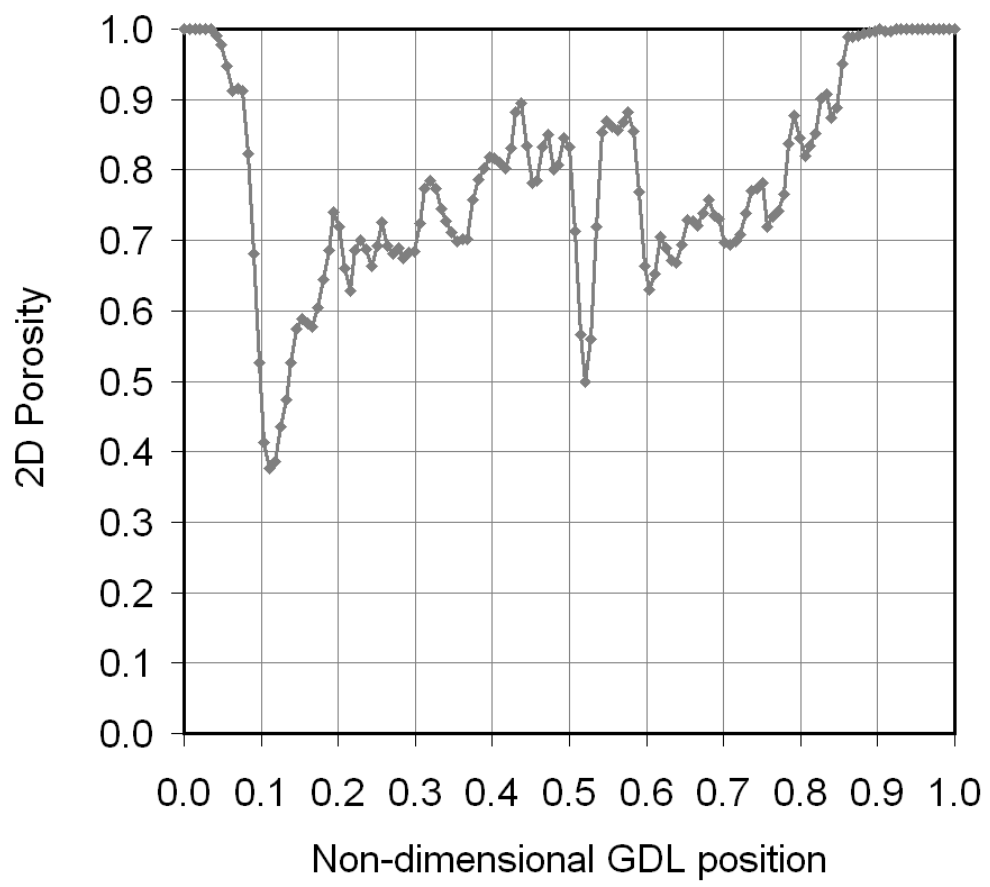

Figure 15. Profile of 2D porosity along the principal flow direction through the GDL.

2D porosity calculated over each $x$-y plane along the z-axis 
What is evidently clear from Figs. 11-14 is that the SPMC-LB technique is capable of correctly predicting whether there is a pressure rise or pressure drop across the cathode GDL for each species constituting a multi-component gas mixture under prescribed fuel cell boundary conditions. The two sets of results from the macro-homogeneous and the LB models however strongly suggests that the heterogeneous porous structure of the GDL does not allow each gas component to establish a linear gradient across its thickness in the z-direction, as suggested by the macro-homogeneous approach. Fundamentally, therefore, the results prove that while a macro-homogeneous approach can adequately capture the general distribution of multi-component gas components through a porous tortuous structure, the true multi-species distribution will be highly dependant upon the actual heterogeneous porous structure. The effect of the heterogeneity of the porous structure can be examined by calculating the percentage difference of the average pressure of each gas as predicted by the two techniques, which is normalised against the non-dimensional thickness of the GDL;

$\Delta_{i}=\left\{\frac{\left.\int_{0}^{1} P_{i}\right|_{L B} \cdot d x-\left.\int_{0}^{1} P_{i}\right|_{G T E} \cdot d x}{P_{\text {tot }}}\right\} \times 100 \%$

The percentage differences for the four test cases including the absolute differences as calculated by the numerator of the bracketed term in Equation 14 are given in Tab. 3.

\begin{tabular}{lcccccccc} 
& \multicolumn{2}{c}{ ADCD } & \multicolumn{2}{c}{ ADCW } & \multicolumn{2}{c}{ AWCD } & \multicolumn{2}{c}{ AWCW } \\
\hline Water & $-558 \mathrm{~Pa}$ & $-0.6 \%$ & $128 \mathrm{~Pa}$ & $0.1 \%$ & $-1.8 \mathrm{kPa}$ & $-1.8 \%$ & $-320 \mathrm{~Pa}$ & $-0.3 \%$ \\
Oxygen & $282 \mathrm{~Pa}$ & $0.3 \%$ & $105 \mathrm{~Pa}$ & $0.1 \%$ & $657 \mathrm{~Pa}$ & $0.7 \%$ & $263 \mathrm{kPa}$ & $0.3 \%$ \\
Nitrogen & $728 \mathrm{~Pa}$ & $0.7 \%$ & $-274 \mathrm{~Pa}$ & $-0.3 \%$ & $2.4 \mathrm{kPa}$ & $2.4 \%$ & $403 \mathrm{~Pa}$ & $0.4 \%$
\end{tabular}

Table 3 Calculated absolute and \% differences between the 3D SPMC-LB and 1D electrochemical modelling approaches 
Table 3 shows that for the carbon paper sample analysed here and the boundary conditions investigated, the maximum difference between the two techniques for the pressure of water vapour based on its thickness-averaged value in absolute terms is $558 \mathrm{~Pa}$. For oxygen, this value is $657 \mathrm{~Pa}$ and for nitrogen is $728 \mathrm{~Pa}$. In percentage terms, therefore, the results also demonstrate that the newlydeveloped SPMC-LB model is capable of accurately predicting the respective pressures of the constituent species; a maximum error of $2.4 \%$ is observed relative to the total pressure in the GDL.

\subsection{Convergence Tests}

The final part of this study focuses on the time that the LB code requires in order to reach a steady-state numerical solution. The accuracy of the numerical results of the LB model depend upon the number of computational timesteps that the code is allowed to perform; increasing the number of timesteps invokes a penalty in terms of computational time. Therefore, a balance has to be established whereby reasonable numerical accuracy is obtained within an acceptable computational timeframe. In the current study, the simulations are carried out on a single core of a quad-core desktop personal computer operating at $2.33 \mathrm{GHz}$ and with $3.25 \mathrm{~GB}$ of RAM. The simulations were allowed to reach 350,000 LB timesteps, which equates to approximately 120 hours of simulation per case. Convergence is tested every 5,000 timesteps commencing from the $10,000^{\text {th }}$ timestep. The error at any given timestep, $n$, is calculated as;

$E_{i, n}=\left.\int_{0}^{1} P_{i}\right|_{L B, n} \cdot d x-\left.\int_{0}^{1} P_{i}\right|_{L B, n-5,000} \cdot d x$

Equation 15

The percentage error for each timestep is defined relative to the $5,000^{\text {th }}$ timestep and can be calculated as; 
$\% \mathrm{E}_{i, n}=\frac{\mathrm{E}_{i, n}}{\mathrm{E}_{i, 10,000}} \times 100 \%$

Figure 16 shows the percentage error as a function of LB timestep for the three gases simulated in test case 2.The results show that by 290,000 timesteps, the percentage error for both water vapour and oxygen have asymptotically reduced below $5 \%$ and are both within $3 \%$ by 335,000 timesteps. The percentage error for nitrogen is relatively higher and falls to $8 \%$ by the $335,000^{\text {th }}$ timestep and reduces to below 7\% after 350,000 timesteps. This is because nitrogen constitutes a larger proportion of the total gas pressure and therefore starts with a larger percentage error which requires a larger number of timesteps to approach a comparably diminutive error. By 350,000 timesteps the actual change in average pressure from timestep-to-timestep for each gas component is around $1 \mathrm{~Pa}$. Therefore, it can be judged that allowing the simulation to continue further will not necessarily yield results that are significantly more accurate. These results therefore suggest that for the image considered in this study, the limit of 350,000 timesteps is sufficient to balance computational time with numerical accuracy.

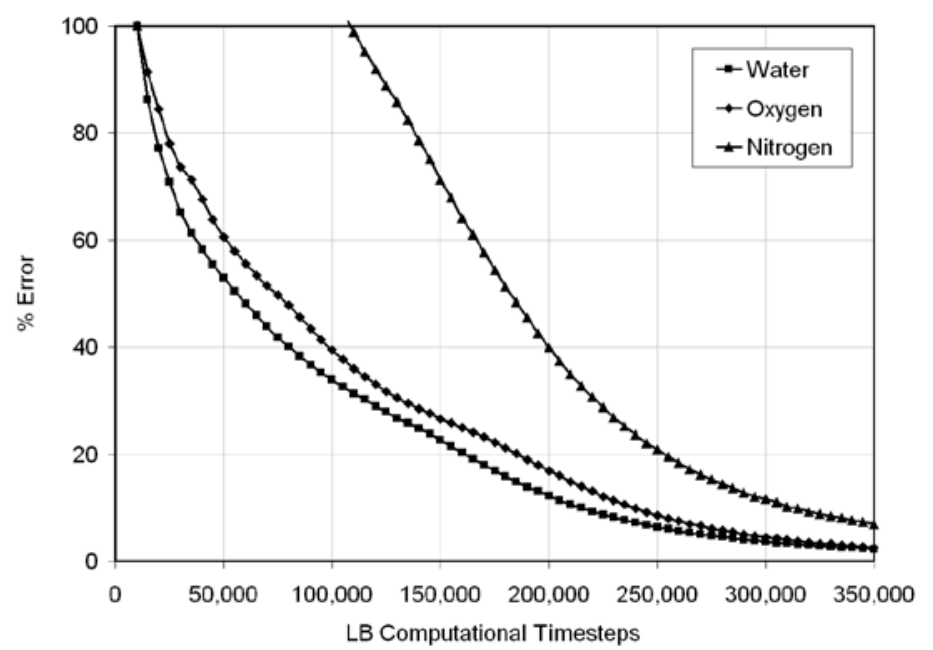

Figure 16. Convergence of lattice Boltzmann simulation results for test case 2

\section{Conclusions}


The current study elucidates the potential to track individual gas species through the heterogeneous porous structure of a fuel cell gas diffusion layer using a combination of X-ray tomography, lattice Boltzmann simulation and electrochemical modelling. Due to the micro-scale characteristic features involved, this has been otherwise formidable as an in-situ characterisation process. The following conclusions can be drawn from the current work:

1. Development of a single-phase multi-component lattice Botlzmann numerical solver: The development of a single-phase multi-component lattice Boltzmann (LB) numerical solver has been presented in this study, which is applied in the D3Q19 scheme with a digitally reconstructed image of a carbon paper fuel cell gas diffusion layer in order to simulate multi-component transport through its heterogeneous porous structure.

\section{Digital reconstruction of a carbon paper fuel cell gas diffusion layer using $X$-ray}

microtomography. The structural binary model employed in the current work is digitally reconstructed using 200 X-ray shadow images taken using X-ray microtomography at a resolution of $1.74 \mu \mathrm{m}$, which are then thresholded and compiled using software techniques. The resulting 3D model employed in the current work for multi-component numerical simulation is 78.3 by 78.3 by $252.3 \mu \mathrm{m}^{3}$.

\section{Merger of a 1D electrochemical model with single-phase multi-component lattice Boltzmann}

numerical solver. Boundary conditions for the LB model are generated using an existing validated 1D electrochemical model which accounts for the structural properties of the fuel cell layers using a macrohomogeneous approach. The predicted pressure profiles are then cross-compared in order to prove that the predicted gradients of each gas component as generated by the LB model agree with that of the electrochemical model, and in order to qualitatively compare the non-linearity of the pressure 
distribution along the principal flow direction. Four fuel cell test cases are selected to test the multicomponent LB model at low current $\left(0.5 \mathrm{~A} / \mathrm{cm}^{2}\right)$; operation with dry anode and dry cathode supplies; dry anode and wet cathode; wet anode and dry cathode and finally wet anode and wet cathode. These test conditions are applied to the electrochemical model in order to determine the boundary conditions at interface $\mathrm{C} 1$ as shown in Fig. 2 as well as the flow rates through the gas diffusion layer.

4. Validation of single-phase multi-component LB model. The simulated results from the LB model show that the SPMC-LB model is capable of correctly simulating the distribution of each species through the heterogeneous structure of the GDL. Furthermore, the results suggest that the actual heterogeneity of the GDL structure in a microscopic 3D LB calculation can induce a variation of up to 2.4\% in the thickness-averaged pressure of an infiltrating species compared to a macroscopic electrochemical 1D calculation for the test conditions considered. It is also shown that for the image size considered in this study, 350,000 computational timesteps are sufficient to obtain a balance between computational time (120 hrs of simulation) and numerical accuracy ( $<1$ Pa pressure variation for each species).

\section{$7 \quad$ Acknowledgements}

This research was supported by the UK Technology Strategy Board (TSB Project No. TP/6/S/K3032H). We acknowledge industrial partners AVL List GmbH, Intelligent Energy Ltd., Johnson Matthey Fuel Cells Ltd., Saati Group Inc., and Technical Fibre Products Ltd. for their support of this work.

\section{Nomenclature}




\section{LB model parameters}

$f_{k}^{i}(\mathrm{x}, t) \quad$ the particle distribution function in the direction of $k$ for species $i$.

$M_{i} \quad$ molecular weight of species $i, \mathrm{~g} / \mathrm{mol}$

$Q^{i i}(\mathrm{x}, t) \quad$ self-collision function between particles of species $i$.

$Q^{i j}(\mathrm{x}, t) \quad$ cross-collision function between particles of species $i$ and species $j$

$t \quad$ time

$\boldsymbol{u}_{i} \quad$ bulk velocity of species $i, \mathrm{~cm} / \mathrm{s}$

$w_{i} \quad$ weighting factor for the D3Q19 LB model.

$\boldsymbol{x} \quad$ spatial position

Greek

$\boldsymbol{\xi}_{k}^{i} \quad$ the particle velocity of species in direction $\mathbf{e}_{k}$ shown in Fig. 3

$\rho_{i} \quad$ density of species $i, \mathrm{~g} / \mathrm{cm}^{3}$

$\tau_{i} \quad$ dimensionless relaxation parameter for self-collision of species $i$.

$\tau_{i j} \quad$ dimensionless relaxation parameter for cross-collision of species $i$ and species $j$.

\section{$1 D$ model parameters}

$c_{i} \quad$ concentration, $\mathrm{mol} / \mathrm{cm}^{3}$

$D_{i j} \quad$ binary species diffusion coefficient, $\mathrm{cm}^{3} / \mathrm{s}$

$D_{i}^{T} \quad$ thermal diffusion coefficient, $\mathrm{cm}^{2} / \mathrm{s}$

I molar flux of water generated in the cathode CL, $\mathrm{mol} / \mathrm{cm}^{2}-\mathrm{s}$

$k_{J} \quad$ permeability pre-factor

K absolute permeability, $\mathrm{cm}^{2}$

$\dot{n}_{i} \quad$ molar flux rate, $\mathrm{mol} / \mathrm{cm}^{2}-\mathrm{s}$

$S_{\text {epf }} \quad$ expanded pore fraction in the quasi-porous PEM 


$$
\begin{array}{ll}
\bar{s}_{i} & \text { molar entropy, } \mathrm{J} / \mathrm{mol}-\mathrm{K} \\
v_{i} & \text { molecular velocity, cm/s }
\end{array}
$$

\section{Greek}

$\begin{array}{ll}\mu_{i} & \text { electrochemical potential, } \mathrm{J} / \mathrm{mol} \\ \mu_{J} & \text { phase viscosity, Pa-s } \\ \xi_{i} & \text { electro-osmotic drag ratio }\end{array}$

\section{Subscripts and superscripts}

$k \quad$ velocity direction in D3Q19 scheme

$\quad$ species $i$, species $j$

J $\quad$ phase $J$

\section{$9 \quad$ References}

${ }^{1}$ CY Wang. Fundamental Models for Fuel Cell Engineering. Chemical Reviews, 2004; 104(10):4727-4766

2 AZ Weber, J Newman. Modeling Transport in Polymer-Electrolyte Fuel Cells. Chemical Reviews, 2004; 104(10):46794726

${ }^{3}$ P Rama, R Chen, R Thring. Polymer Electrolyte Fuel Cell Transport Mechanisms: A Universal Modelling Framework from Fundamental Theory. Proc. IMechE Part A: J. Power and Energy, 2006; 220: 535-550

${ }^{4}$ P Rama, R Chen. Polymer Electrolyte Fuel Cell Transport Mechanisms: A Universal Approach to Multi-Layer TwoPhase Modelling through the General Transport Equation. ASME J. Fuel Cell Sci. Technol., 2010; DOI: $10.1115 / 1.4001004$

${ }^{5}$ T Springer, T Zawodzinski, S Gottesfeld. Polymer Electrolyte Fuel Cell Model. J. Electrochem. Soc., 1991; 138: 23342342

${ }^{6}$ D Bernardi, M Verbrugge. A Mathematical Model of the Solid-Polymer-Electrolyte Fuel Cell. J. Electrochem. Soc., 1992; 139:2477-2491

7 TF Fuller, J Newman. Water and Thermal Management in Solid-Polymer-Electrolyte Fuel Cells. J. Electrochem. Soc., 1993; 140(5):A1218-1225

${ }^{8}$ D Natarajan, T Van Nguyen. A Two-Dimensional, Two-Phase, Multi-Component, Transient Model for the Cathode of a Proton Exchange Membrane Fuel Cell Using Conventional Gas Distributors. J. Electrochem. Soc., 2001; 148(12): A1324-A1335

${ }^{9}$ P Berg, K Promislow, J St. Pierre, J Stumper, B Wetton. Water management in PEM Fuel Cells. J. Electrochem. Soc., 2004; 151(3): A341-A353

${ }^{10}$ E Bergisson, M Noponen, M Vynnycky. Analysis of Two-Phase Non-Isothermal Model for a PEFC. J. Electrochem. Soc., 2005; 152(5): A1021-A1034

${ }^{11}$ GJM Janssen. A Phenomenological Model of Water Transport in a Proton Exchange Membrane Fuel Cell. Journal of The Electrochemical Society, 2001; 148(12):A1313-A1323 
${ }^{12}$ P Zhou, CW Wu. Numerical Study on the Compression Effect of Gas Diffusion Layer on PEMFC Performance. J. Power Sources, 2007; 170: 93-100

13 AZ Weber, J Newman. Transport in Polymer-Electrolyte Membranes II. Mathematical Model. Journal of The Electrochemical Society, 2004; 151(2):A311-A325

${ }^{14}$ AZ Weber, J Newman. A Theoretical Study of Membrane Constraint in Polymer-Electrolyte Fuel Cells. AIChE Journal, 2004; 50(12):3215-3226

${ }^{15}$ AZ Weber, J Newman. Effects of Microporous Layers in Polymer Electrolyte Fuel Cells. J. Electrochem. Soc., 2005; 152(4):A677-A688

${ }^{16}$ E Hontanon, MJ Escudero, C Bautista, PL Garcia-Ybarra, L Daza. Optimisation of flow-field in polymer electrolyte membrane fuel cells using computational fluid dynamics techniques. J. Power Sources, 2000; 86:363-368

${ }^{17} \mathrm{G}$ Hu, J Fen, S Chen, Y Liu, K Cen.Three-dimensional numerical analysis of proton exchange membrane fuel cells (PEMFCs) with conventional and interdigitated flow fields. J Power Sources, 2004; 136:1-9

${ }^{18} \mathrm{ZH}$ Wang, CY Wang, KS Chen. Two-phase flow and transport in the air cathode of proton exchange membrane fuel cells. J. Power Sources, 2001; 94: 40-50

${ }^{19}$ U Passaogullari, CY Wang. Two-Phase Modeling and Flooding Prediction of Polymer Electrolyte Fuel Cells. J. Electrochem. Soc., 2005; 152(2):A380-A390

${ }^{20} \mathrm{H}$ Ju, CY Wang. Experimental Validation of a PEM Fuel Cell Model by Current Distribution Data. J. Electrochem. Soc., 2004; 151(11):A1954-A1960

${ }^{21}$ AP Manzo, FF Marzo, AR Pierna, J Barranco, A Lorenzo, J Barroso. Design Optimization of a Polymer Electrolyte Membrane Fuel Cell, PEMFC. J. New Mater. Electrochem. Syst., 2009; 12: 133-137

${ }^{22}$ R Theidmann, F Fleisher, C Hartnig, W Lehnert, V Schmidt. Stochastic 3D Modeling of the GDL Structure in PEMFCs Based on Thin Section Detection. J. Electrochem. Soc., 2008; 155(4):B391-B399

${ }^{23}$ PK Sinha, PP Mukherjee, CY Wang. Impact of GDL structure and wettability on water management in polymer electrolyte fuel cells. J Mater Chem., 2007; 17: 3089-3103

${ }^{24} \mathrm{~J}$ Becker, V Schulz, A Weigmann. Numerical Determination of two-phase material parameters of a gas diffusion layer using tomography images. ASME J. Fuel Cell Sci. Technol., 2007; 5(2):75-89

${ }^{25}$ P Rama, Y Liu, R Chen, H Ostadi, K Jiang, X Zhang, R Fisher, M Jeschke. An X-Ray Tomography Based LatticeBoltzmann Simulation Study on Gas Diffusion Layers of Polymer Electrolyte Fuel Cells. ASME J. Fuel Cell Sci. Technol., article in press

${ }^{26}$ S Arcidiacono, IV Karlin, J Mantzaras, CE Frouzakis. Lattice Boltzmann model for the simulation of multicomponent mixtures. Physical Review E, 2007; 76(046703):1-11

${ }^{27}$ AS Joshi, KN Grew, AA Peracchio, WKS Chiu. Lattice Boltzmann modeling of 2D gas transport in a solid oxide fuel cell anode. J. Power Sources, 2007; 164(2):631-638

${ }^{28}$ WKS Chiu, AS Joshi, KN Grew. Lattice Boltzmann model for multi-component mass transfer in a solid oxide fuel cell anode with heterogeneous internal formation and electrochemistry. Eur. Phys. J. Special Topics, 2009; 171:159-165

${ }^{29}$ LS Luo, SS Girimaji. Theory of the lattice Boltzmann method: Two-fluid model for binary mixtures. Physical Review E, 2003; 67(3):036302

${ }^{30}$ XX Zhang, JW Crawford, AG Bengough, IM Young. On boundary conditions in the lattice Boltzmann model for advection and anisotropic dispersion equation. Advances in Water Resources, 2002; 25(6):601-609

${ }^{31} \mathrm{H}$ Ostadi, K Jiang, PD Prewett. Micro/nano X-ray tomography reconstruction fine tuning using scanning electron microscope images. Micro and Nano Letters, 3(4):106-109

32 H Ostadi, P Rama, Y Liu, R Chen, X Zhang, K Jiang, Nanotomography based study of gas diffusion layers.Microelectronic Engineering, 2010; (Article in press)

${ }^{33}$ SkyScan. CT Analyser Users Guide. http://www.skyscan.be/next/CTan_UserManual.pdf (last accessed 18th Nov 2009) 